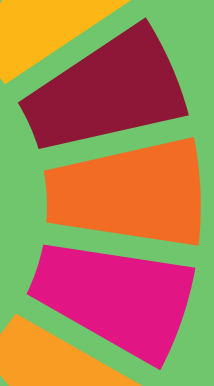

\title{
LUCES Y SOMBRAS \\ PARA EL DESARROLLO \\ DE UNAS FINANZAS \\ SOSTENIBLES EN \\ EL SECTOR BANCARIO \\ EUROPEO
}

\section{OPPORTUNITIES}

AND CHALLENGES

FOR SUSTAINABLE FINANCE IN THE

\section{EUROPEAN BANKING}

\section{INDUSTRY}

Pablo Esteban-Sánchez.

estebanpab@gmail.com

Raquel Arguedas Sanz

rarguedas@cee.uned.es

Cristina Ruza Paz-Curbera

cruza@cee.uned.es

Grupo de Investigación UNED-FINRES 


\section{RESUMEN}

La Unión Europea y las autoridades de supervisión de su industria bancaria han desarrollado en la última década relevantes iniciativas para el impulso de las finanzas sostenibles con el fin de facilitar la transición hacia una economía más resiliente, climáticamente neutra y alineada con los objetivos adoptados en la Agenda 2030 y el Acuerdo de París. A pesar de ello existen importantes desafíos latentes en el ámbito regulatorio, de supervisión y operativo. Este artículo, a partir de las iniciativas más relevantes, analiza los principales desafíos concluyendo que los más importantes se refieren, entre otros, a la implicación a largo plazo de accionistas e inversores, la aplicación de la taxonomía europea recientemente aprobada, la calidad y acceso a la información ASG para la inversión en actividades sostenibles, la reducción del green y social washing, la gestión y análisis de los riesgos ASG, y el desarrollo y etiquetado de productos de financiación e inversión sostenibles para el segmento minorista. Las conclusiones son útiles para gestores, reguladores e investigadores con el fin de diseñar acciones y estudios que posibiliten una eficaz implementación de las iniciativas y regulaciones en materia de finanzas sostenibles.

Palabras clave: Finanzas sostenibles; Finanzas verdes; ODS; ASG; Inversión sostenible; Inversión de impacto; inversiones verdes; Banca; Índices de referencia.

\section{ABSTRACT}

Over the last decade, the European Union and the European Banking Supervisory Authorities have carried out relevant initiatives to develop sustainable finance aimed at facilitating the transition towards a more sustainable and climate-neutral economy aligned with the objectives of the 2030 Agenda and Paris Agreement. In spite of these efforts, there are still significant regulatory, supervisory and operational questions to deal with. This article, based on the most relevant initiatives, analyses the major operational challenges, which are related to the long-term shareholders and investors engagement, the difficulties to adopt the recently approved European taxonomy, the quality and availability of ESG information for investing in green and sustainable activities, the green and social washing, the ESG risks integration and analysis, and the development of sustainable finance and retail investment products. The conclusions would be useful for managers, regulators and researchers whose aims include taking actions and enabling the effective implementation of initiatives and regulations on sustainable finance.

Keywords: Sustainable finance; Green finance; SDG; ESG; Sustainable investment; Impact investment, Green investments; Banking industry; Benchmarks.

Pablo Esteban-Sánchez. Investigador vinculado al Grupo UNED-FINRES. Doctor en Economía Financiera de la Empresa por la UCM. Profesor asociado a la Universidad Europea de Madrid. Sus líneas de investigación giran en torno al desarrollo de las finanzas sostenibles y factores ASG, la relación entre la RSC y la rentabilidad en la industria financiera, y la RSC en las PYMEs. Cuenta con publicaciones en revistas académicas internacionales y participaciones en Congresos internacionales. Compagina su labor académica con una larga actividad profesional en finanzas internacionales y banca de desarrollo en América Latina. 
Raquel Arguedas Sanz. Investigadora vinculada al Grupo UNED-FINRES. Profesora Titular de Economía Financiera de Universidad Nacional de Educación a Distancia (UNED). Doctora en Ciencias Económicas y Empresariales por la UNED. Premio Extraordinario de Doctorado y Premio Víctor Mendoza 2010 del Instituto de Estudios Económicos. Acreditada con la certificación Financial Risk Manager ${ }^{\mathrm{TM}}$ de la Global Association of Risk Professionals. Áreas centrales de investigación y publicación: Finanzas en la empresa, gestión y supervisión de riesgos financieros en banca, Finanzas Sostenibles.

Cristina Ruza Paz-Curbera. Investigadora vinculada al Grupo UNED-FINRES. Profesora Contratada Doctora del Departamento de Economía Aplicada en la UNED. Secretaria del Máster en Sostenibilidad y RSC. Economista con experiencia en el sistema financiero, comprometida con la investigación de un nuevo modelo económico que responda no sólo a los criterios tradicionales de rentabilidad, sino a nuevos criterios ligados a la sostenibilidad.

\section{INTRODUCCIÓN}

La toma de conciencia de la emergencia climática derivada del calentamiento global del planeta, la crisis financiera internacional del 2008 y la actual crisis social y económica en la que nos vemos inmersos por la pandemia del Covid-19, han hecho más evidente la necesidad de integrar a nivel global las consideraciones medioambientales, sociales y de gobernanza (factores ASG) para construir un modelo de economía más sostenible, robusto y resiliente, a la vez que menos frágil a futuras crisis o nuevos cisnes "verdes" y "negros". El logro de ello dependerá en gran medida de la coordinación internacional, del sendero que se paute para dicha transición y de la apuesta decidida por la innovación tecnológica y el avance científico.

Previamente como antesala a todo ello, amerita citar en este preámbulo el debate y las valiosas aportaciones quedistintos autores realizaronen losúltimos 20 años del sigloXXentornoal constructo de la Responsabilidad Social Corporativa (RSC) acuñado por Bowen (1953) en su libro "Responsabilidades Sociales del hombre de negocios", y al dilema de cómo lograr un equilibrio equitativo y sostenible mencionado anteriormente por Abrams (1951). Contribuciones relevantes, entre otras tales como la Teoría de la actuación social de la empresa o corporate social performance (Carroll, 1979, 1981, 1991), la Teoría de los stakeholders o de los grupos o partes implicadas (Emshoff y Freeman, 1978; Freeman, 1984; Evan y Freeman, 1988; Freeman, 1994), y la Teoría de la buena ciudadanía empresarial (Carroll, 1999; Wood y Logsdon, 2002).

Asimismo nos parece relevante mencionar el Pacto Mundial de las Naciones Unidas (1999), y las referencias a la integración de las consideraciones a largo plazo de tipo social, medioambientales y económicas para un Desarrollo Sostenible realizadas por la World Commission on Environment and Development (1987). EI propio Tratado de la Unión Europea (UE) en sus artículos 3.3 y 21 hace hincapié en las cuestiones sociales y medioambientales y no sólo económicas del desarrollo sostenible.

Bajo este contexto, durante las dos últimas décadas se intensificaron las iniciativas internacionales para una transición hacia una economía y un desarrollo más sostenible e inclusivo, que recupere el equilibrio entre los sistemas económicos, sociales y ecológicos. Las iniciativas han ido evolucionando desde las recomendaciones, estándares y/o principios de adhesión voluntaria, a directivas y reglamentos que obligan a entidades y empresas de ciertos sectores y/o tamaño en ámbitos diversos relativos a la consideración de los factores ASG. El anexo 1 recoge en una Tabla un resumen de las iniciativas más relevantes.

Pero en un contexto más reciente que sirve de marco para este artículo, dos iniciativas internacionales están marcando el punto de referencia para la UE en la transición hacia un nuevo modelo más sostenible: La Agenda 2030 para el Desarrollo Sostenible de las Naciones Unidas y el Acuerdo de París para el Cambio Climático. 
En este contexto resulta prioritario el desarrollo de unas finanzas sostenibles con el fin de canalizar adecuadamente la financiación e inversión hacia inversiones más sostenibles y alineadas con los Objetivos de Desarrollo Sostenible (ODS) señalados en la Agenda 2030 y con la transición hacia una economía neutra de emisiones de carbono que permita alcanzar las metas fijadas en el Acuerdo de París. Estos objetivos no están exentos de dificultades, derivadas de la complejidad de comprender, medir, gestionar e integrar adecuadamente las consideraciones y riesgos ambientales, sociales y de gobernanza (riesgos ASG) en las decisiones de financiación e inversión y los mecanismos que regulan la estabilidad financiera. Estos retos unidos a la urgencia de establecer Planes de Acción actuaron de llamada de atención para que la UE, los reguladores y supervisores de la industria financiera y los bancos centrales unieran sus fuerzas y pusieran en marcha una agenda completa de políticas y planes de acción para el impulso de las finanzas sostenibles.

Así la Comisión Europea (CE) en marzo del 2018 recogió las conclusiones del informe final del Grupo Experto de Alto Nivel en Finanzas Sostenibles (EU High-Level Expert Group on Sustainable Finance, 2018) y publicó el Plan de Acción: Financiar el desarrollo sostenible (Comisión Europea, 2018) estableciendo una estrategia de la UE sobre finanzas sostenibles y una hoja de ruta que guiara el trabajo futuro en todo el sistema financiero.

En diciembre del 2019 se publicó una Comunicación de la CE (COM) con "El Pacto Verde Europeo" (Comisión Europea, 2019a) que constituye la hoja de ruta para dotar a la UE de una economía sostenible y asumir un papel de liderazgo al comprometerse con el objetivo de una economía libre de emisiones netas de gases de efecto invernadero en 2050.

Y el 14 de enero del 2020 hizo público el Plan de Inversiones para una Europa Sostenible. Plan de Inversiones del Pacto Verde Europeo (Comisión Europea, 2020a) donde estima que la consecución de las metas climáticas y energéticas para 2030 exigirá inversiones adicionales de 260000 millones de euros anuales en la década 2021-2030. Para ello el Plan de Inversiones movilizará al menos 1 billón de euros en la próxima década, de los que 503.000 millones se movilizarán contra el presupuesto de la UE, y otros 279.000 millones a través del sistema de garantías de la UE dentro del Programa InvestEU. Además habrá una cofinanciación nacional de los Estados miembros de la UE por valor de 114.000 millones de euros, y al menos 25.000 millones de euros provenientes de los Fondos de Innovación y Modernización del régimen de subastas de los derechos de emisión de carbono. Se reservarán 100.000 millones de euros (2021-2027) para ayudar a las regiones más afectadas mediante el denominado Mecanismo para una Transición Justa. Un mínimo por tanto del 25\% del Presupuesto de la UE se destinará a objetivos climáticos. Además el Banco Europeo de Inversiones (BEI) destinará en torno a 600000 millones de euros en dicha década para la financiación de la acción por el clima y la sostenibilidad medioambiental hasta llegar al 50\% de su actividad crediticia en el 2050.

Este artículo se focaliza en la importancia del sector bancario y financiero en Europa para canalizar los flujos financieros y de inversión hacia actividades y proyectos que contribuyan al logro de estos objetivos, y realiza un análisis de las palancas y desafíos que el sector tiene en la actualidad para un eficaz y decidido desempeño en esta labor.

En un primer apartado se realiza una revisión de las principales palancas que tiene el sector bancario europeo para el impulso de las finanzas sostenibles y se clasifican en los ámbitos regulatorio, gestión y análisis de riesgos ASG y, finalmente, innovación de productos de financiación y de inversión sostenibles. En un segundo apartado se abordan los desafíos más importantes que el sector debe afrontar para el mejor desarrollo de la financiación e inversión sostenible. En un tercer apartado se aportan unas conclusiones y futuras líneas de investigación. Y finalmente se recoge la bibliografía y el anexo. 


\section{PRINCIPALES PALANCAS EN EL SECTOR BANCARIO EUROPEO PARA EL DESARROLLO DE LAS FINANZAS SOSTENIBLES}

\section{1. ÁMBITO REGULATORIO}

Si bien el impulso regulatorio alcanza países y regiones de todo el mundo, este artículo se va a centrar exclusivamente en el ámbito de la UE.

\subsubsection{IMPLICACIÓN ACCIONARIAL A LARGO PLAZO (ENGAGEMENT)}

La Directiva UE 2017/828 sobre el fomento de la implicación a largo plazo de los accionistas (Parlamento Europeo y Consejo de la UE, 2017) exige que la política de engagement describa, entre otros, el seguimiento que realizan los inversores a las compañías tanto en su desempeño financiero como no financiero; cómo sus estrategias accionariales consideran su responsabilidad legal y cómo van a incentivar y supervisar que los gestores de activos sigan estas directrices.

\subsubsection{TAXONOMÍA VERDE}

El Reglamento UE 2020/852, también conocido como Reglamento de la Taxonomía (Parlamento Europeo y Consejo de la UE, 2020) define un concepto común de «inversión medioambientalmente sostenible» y los criterios de selección de actividades económicas en función de su contribución al logro de seis objetivos medioambientales. Los criterios técnicos de selección para los dos primeros objetivos (mitigación y adaptación al cambio climático) entran en vigor a finales del 2021 mientras los relativos a los cuatro objetivos ambientales restantes todavía no se han definido ${ }^{1}$.

El Reglamento se complementa con un anexo técnico donde se especifican los criterios para la contribución a los dos primeros objetivos medioambientales, el uso de la nomenclatura NACE para las actividades económicas, los criterios de clasificación de amenazas climáticas por sectores y las matrices sectoriales de sensibilidad ante amenazas climáticas.

\subsubsection{ESTÁNDARES Y ETIQUETAS}

La Iniciativa Financiera dentro del Programa de Naciones Unidas para el Medio Ambiente y la Federación Bancaria Europea (UNEP Finance Initiative y European Banking Federation, 2021) recomiendan el desarrollo de etiquetas verdes desde el momento de su contratación por el segmento minorista. En la misma línea, la International Platform on Sustainable Finance en su Informe Anual del 2020 (IPSF, 2020) apoya el uso de determinados estándares internacionales como los principios para bonos verdes (GBP) o sociales (SBP).

El Grupo Técnico de Expertos en Finanzas Sostenibles (TEG) publicó en 2019 una propuesta de estándar para un Bono Verde Europeo (EU Technical Expert Group on Sustainable Finance, 2019), base para la futura Ley del Bono Verde Europeo.

\footnotetext{
${ }^{1}$ Entrará en vigor a finales de 2022.
} 
Asimismo, el BEl está desarrollando un préstamo verde y un bono verde, ambos referenciados al GBSEU, mientras que el Programa y el Fondo InvestEU otorgarán garantías para proyectos de inversión verdes.

Además, la UE está considerando elaborar un marco regulatorio para el desarrollo de una EcoLabel Europea² para productos de inversión verdes minoristas.

Para todos estos estándares o etiquetas verdes, el Reglamento de la Taxonomía de la UE requiere que estén alineadas con dicha taxonomía.

\subsubsection{MEDICIÓN, REPORTE Y TRANSPARENCIA}

En Europa, grandes compañías cotizadas, bancos y aseguradoras, entre otras de interés público, deben cumplir con la Directiva 2014/95 (NFRD) sobre reporte de información no financiera (Parlamento Europeo y Consejo de la UE, 2014). Esta Directiva fue completada por las COM de "Directrices sobre la presentación de informes no financieros" (Comisión Europea, 2017) y las "Directrices sobre la presentación de informes no financieros: Suplemento sobre la información relacionada con el clima" (Comisión Europea, 2019b), que establecen criterios de materialidad financiera, así como materialidad social y medioambiental. La CE estima que 6.000 empresas deberán cumplir la norma, una cifra que en España puede llegar hasta las 600 compañías.

Por su parte el Consejo de Estabilidad Financiera (FSB), impulsó el Grupo de Trabajo sobre divulgación de información financiera relacionada con el cambio climático, que publicó el "Informe sobre divulgación de información financiera relacionada con el cambio climático" (Task Force on Climate-related Financial Disclosures, 2017).

El Reglamento UE 2019/2088 (SFDR) de divulgación de información sobre sostenibilidad en el sector financiero, (Parlamento Europeo y Consejo de la UE, 2019a) establece obligaciones para los participantes de la industria financiera.

Y el Reglamento de la Taxonomía refuerza las obligaciones de transparencia del SFDR respecto a la información precontractual y periódica de los productos e inversiones verdes, y de la NFRD sobre las actividades económicas medioambientalmente sostenibles.

La propuesta de estándar de Bono Verde Europeo establece la obligación de un informe de impacto durante la vida del bono cuya verificación recomienda y de informes anuales de asignación de fondos.

Finalmente, la Autoridad Europea de Valores y Mercados (ESMA) ha propuesto desarrollar una regulación de requerimientos de divulgación para las Agencias de Calificación (European Securities and Markets Authority, 2019) y las autoridades europeas de supervisión del sector financiero (ESAs) ${ }^{3}$ han elaborado un borrador del Reglamento de Normas Técnicas Regulatorias (Joint Committee of the European Supervisory Authorities, 2021).

\footnotetext{
${ }^{2}$ Similar a la etiqueta Nordic Swan que tienen algunos países nórdicos ${ }^{3}$ EBA, ESMA y EIOPIA
} 


\subsubsection{CONSTRUCCIÓN DE ÍNDICES DE REFERENCIA O BENCHMARKS}

El Reglamento (UE) 2019/2089 (Parlamento Europeo y Consejo de la UE, 2019b) obliga a los administradores de los índices de referencia climáticos de la UE a publicar información sobre metodologías, métricas, ponderaciones, selección y exclusión de activos y alineamiento con el Acuerdo de París, así como la frecuencia y procedimiento de sus revisiones. Se establecen distintas fases para la entrada en vigor según el tipo de la información a divulgar, desde abril de 2020 al 31 de diciembre del 2022.

\subsection{GESTIÓN Y ANÁLISIS DE RIESGOS ASG}

El Consejo de Estabilidad Financiera del G20 en abril de 2015 señaló que los riesgos del cambio climático pueden ser sistémicos para la estabilidad financiera global.

Tales efectos potencialmente sistémicos motivan que se hayan generado expectativas sobre la incorporación de riesgos ASG en el pilar 1 de Basilea III (BIS III). En esta línea, la Autoridad Bancaria Europea (EBA), siguiendo lo encomendado en los artículos 501c de la Regulación de Requerimientos de Capital) y 98 (8) de la Directiva de Requerimientos de Capital modificados en el 2019, (Parlamento Europeo y Consejo de la UE, 2019 c, d), está trabajando en un Informe para la gestión y supervisión de riesgos ASG (European Banking Authority, 2020), cuyas conclusiones se publicarán en junio de 2021.

El Banco Central Europeo (2020) publicó una "Guía de referencia sobre riesgos relacionados con el clima y medioambientales" para recoger las expectativas supervisoras en materia de gestión y comunicación de estos riesgos.

Y la CE publicó un estudio elaborado por BlackRock Financial Markets Advisory (2020) para la integración de factores ASG en el marco prudencial bancario de la UE.

El sector bancario debe entonces estar preparado para desarrollar Sistemas Análisis de Riesgos Ambientales y Sociales (ARAS), y en un futuro incorporar escenarios de riesgos climáticos en las pruebas de estrés financiero.

\subsection{INNOVACIÓN Y DESARROLLO DE PRODUCTOS DE FINANCIACIÓN E INVERSIÓN SOSTENIBLES}

Las evidencias indican que, como consecuencia del Covid-19, el mercado de Inversión Socialmente Responsable (ISR) ha estimulado el crecimiento de bonos sociales, bonos sostenibles/ODS, bonos temáticos y, más recientemente, bonos pandémicos. La CE ha anunciado que el Instrumento Europeo de Apoyo Temporal para Atenuar los Riesgos de Desempleo en una Emergencia (SURE) se emitirá como bonos sociales alineados con los SBP.

El Informe Anual del Observatorio Español de la Financiación Sostenible (2020) destaca que en España 2020 las emisiones de bonos verdes, sociales y sostenibles en 2020 han aumentado un $54 \%$ respecto a 2019. Los bonos sociales son los que alcanzaron el mayor crecimiento con un $88 \%$, seguido de los bonos sostenibles con un $62 \%$. 
Respecto a los productos de financiación verde o sostenible, la IPSF en el ya mencionado Informe Anual del 2020 indica que las principales innovaciones son los préstamos verdes (GL), los préstamos vinculados a la sostenibilidad (SLL) y los bonos vinculados a la sostenibilidad (SLB). Gracias a este desarrollo, la financiación sostenible resulta más accesible para empresas más pequeñas o incluso sectores de actividad económica que no participan activamente en el mercado de bonos. Los SLL y los SLB son productos cuyos fondos son destinados a fines corporativos generales, pero la tasa de interés varía según el logro de unos objetivos de desempeño predeterminados. En 2019 el mercado SLL alcanzó 122.000 millones de dólares americanos a nivel mundial. El mercado europeo lidera el crecimiento con más del $80 \%$ de la actividad global de SLL. En España los SLL movilizaron 18.002 millones en 2020, un 38\% más que en el 2019, siendo un 50\% para proyectos sostenibles, seguidos de un $36 \%$ en préstamos ligados a la sostenibilidad, un $9 \%$ en préstamos verdes con certificación y el resto agrupados en préstamos sociales.

Por el lado de la Inversión de Impacto, destacar los fondos institucionales de inversión de impacto que compaginan rentabilidad financiera con la generación y medición de impacto social. Pero las innovaciones más relevantes se adscriben a los mecanismos de contratación público-privada (Contratos de Impacto Social y Contratos de Pago por Resultados, mal llamados bonos de impacto) que se apoyen en estructuras vehículo de inversión dentro de estrategias de financiación combinada (blended finance), y de colaboración públicoprivada con mecanismos para el reparto y transferencias de riesgos y rentabilidades, responsabilidades de gestión y costes de transacción.

Finalmente, hay que destacar algunas iniciativas de democratización de la inversión de impacto como el crowdfunding y crowddlending de impacto y algunos ejemplos de renting, factoring y confirming sostenibles.

\section{PRINCIPALES DESAFÍOS EN EL SECTOR BANCARIO EUROPEO PARA EL DESARROLLO DE UNAS FINANZAS SOSTENIBLES}

\section{1. ÁMBITO REGULATORIO}

El impulso regulatorio es notable como hemos señalado en el apartado anterior pero existen importantes retos que afrontar dentro de las fronteras de la UE.

\subsubsection{IMPLICACIÓN ACCIONARIAL A LARGO PLAZO (ENGAGEMENT)}

El Fondo Mundial para la Naturaleza (2020) propone impulsar estrategias que trasciendan a los inversores institucionales y gestores de activos. Primeramente promueve un engagement del sector financiero con las empresas de su cartera de préstamos y no sólo de inversión, subraya la necesidad de estrategias y mecanismos de transición para favorecer la implicación en las empresas más intensivas en carbono, y una acción coordinada que contribuya a un engagement colectivo y público también para inversores minoristas.

Es necesario también ampliar el engagement a las pequeñas y medianas empresas (PYMEs) que no están sujetas a la NFRD y SFDR y cuyo cumplimiento les resultaría muy oneroso, y que hoy afrontarían su implicación desde una óptica voluntaria. La labor de asesoramiento a inversores minoristas y PYMEs que podrían realizar entidades financieras podría contribuir a dicho objetivo. 


\subsubsection{TAXONOMÍA}

Los desafíos para la aplicación del Reglamento de la Taxonomía son muchos y no exentos de complejidad. En primer lugar, por el momento no requiere que los inversores busquen verificación externa, si bien la CE revisará este punto para el año 2022.

La UNEPFI y la Federación Bancaria Europea (2021) apuntan a la falta de calidad y disponibilidad de datos sobre PYMEs y activos radicados fuera de la UE, la carencia de herramientas tecnológicas y metodologías comunes que faciliten la evaluación de la adaptación al cambio climático de los activos, y la falta de alineación con otras clasificaciones de actividades utilizadas en otros países con distintos alcances y precisiones. En este último sentido, International Platform on Sustainable Finance (2020) anuncia que a mediados del 2021 publicará un Informe para una Taxonomía Común. El verdadero reto consistirá en combinar una estandarización con la flexibilidad y adaptación a las distintas urgencias según geografías.

Otras dificultades señaladas son la heterogeneidad internacional de la nomenclaturas de las actividades económicas utilizadas distintas a la NACE utilizada por la taxonomía, por ejemplo la nomenclatura ISIC (Naciones Unidas, 2008), la imposibilidad de delimitar el uso de los fondos porque la mayor parte de los préstamos de los bancos se conceden para propósitos generales, y la existencia de muchas empresas con actividades múltiples por lo que clasificarlos bajo la NACE no es sencillo. También apuntan al enorme esfuerzo y recursos de tiempo, gestión de documentación, adaptación de los sistemas de información y control y una importante brecha entre las prácticas internas existentes y el marco de la taxonomía de la UE que acusa deficiencias de diseño para adecuarse al segmento minorista y de las PYMEs, y que se aplicaría mejor a un ámbito general o sectorial.

Por ello urge la capacitación a equipos internos en la banca expertos en la taxonomía y cuestiones ASG, servicios externos de verificación y certificación, y herramientas o mapas de alineación con estándares o clasificaciones preexistentes.

Finalmente mencionar el alcance limitado de la Taxonomía de la UE que sólo hace referencia a la Taxonomía de actividades climáticas. La CE espera que a finales de 2021 se aborden los complejos trabajos para desarrollar una taxonomía social. El propio TEG en su Informe final del 2020 así lo recomendaba teniendo en cuenta que los ODS tienen una fuerte dimensión social y la interrelación existente entre lo verde y lo social. En este contexto, el concepto de la Inversión de Impacto deberá ser integrado en el Plan de Acción para las Finanzas Sostenibles. Estas inversiones según la Global Impact Investing Network (GIIN) ${ }^{4}$ son aquellas que buscan un impacto social y ambiental positivo y mensurable conjuntamente con una rentabilidad financiera. La taxonomía tampoco incluye a las actividades que dañan de forma significativa el medioambiente, más conocidas como "marrones". EI TEG también recomienda desarrollar una Taxonomía marrón que valore la reducción de los impactos negativos medioambientales e impulse la transición ecológica.

\subsubsection{ESTÁNDARES Y ETIQUETAS}

Los desafíos son también numerosos. Primeramente, no hay homogeneidad internacional en los estándares y etiquetas de productos financieros e inversiones verdes o sostenibles, lo cual genera confusión en los inversores que operan en mercados globales.

${ }^{4}$ Ver definición en https://thegiin.org/impact-investing/need-to-know/\#what-is-impact-investing 
Tampoco hay en Europa un sistema de incentivos fiscales para la Inversión sostenible, ni incentivos desde la óptica de la supervisión y la óptica prudencial en materia, por ejemplo, de requerimientos de capital en función del riesgo. EI TEG en su propuesta de un GBS apunta a la necesidad de estudiar por parte de las autoridades bancarias la viabilidad de estos incentivos.

A lo expuesto se suma que la Taxonomía UE no tiene como objetivo prioritario el desarrollo de un etiquetado para productos. Persigue fijar criterios para clasificar las actividades sostenibles y reforzar la SFDR en lo que respecta a la transparencia de información pre-contractual y periódica en la SFDR. La honrosa excepción está en la ley del Bono Verde Europeo y las menciones a estudiar la posibilidad futura de los EColabels para productos en el segmento minorista. Ni tan siquiera se habla de registros, por ejemplo, para fondos ISR. El problema una vez más reside en que para un etiquetado diligente habría que estar en disposición de medir el impacto y todavía existen grandes retos para la aplicación de unas métricas alineadas con los ODS y el Acuerdo de París.

\subsubsection{MEDICIÓN, REPORTE Y TRANSPARENCIA}

A pesar de haber experimentado un gran avance regulatorio, los desafíos son importantes en materia de divulgación y transparencia. En primer lugar hay una diversidad de guías o estándares para el reporte de memorias de sostenibilidad, lo que dificulta la comparabilidad. Por ello, cinco organizaciones de referencia, el Carbon Disclosure Project (CDP), Climate Disclosure Standards Board (CDSB), GRI, International Integrated Reporting Council (IIRC) y el Sustainability Accounting Standards Board (SASB), publicaron en diciembre de 2020 una propuesta de estándar de divulgación financiera relacionada con el clima (Carbon Disclosure Project et al 2020).

A ello se une la diversidad de metodologías e indicadores de medición del impacto medioambiental y climático sin que ninguna se haya convertido en el referente universal. Coexisten diversas metodologías que miden la huella de carbono y múltiples propuestas de indicadores y métricas a utilizar realizadas en el marco de la reglamentación de la UE. Asimismo la diversidad de prácticas de gestión y medición de impacto social explica iniciativas como el Impact Management Project (IMP) ${ }^{5}$ y el IRIS ${ }^{6}{ }^{6}$ que tratan de alinear métricas con los ODS y acercarlas a las PYMEs. El IMP es impulsado por importantes referentes como GIIN, GRI, el IR, el CDP, CDSB, PRI, SASB, UNEP FI, OECD, IFC, NU y otros. Existe también una diversidad de metodologías con resultados dispersos y poco correlacionados aplicadas por las CRA (Berg et al., 2019) lo que genera distorsiones en las calificaciones de un mismo activo y en las decisiones de los inversores. Así lo ha puesto de manifiesto la ESMA en su reciente carta a la CE (European Securities and Markets Authority, 2021).

Destacar la falta de acceso y calidad de los datos de muchas empresas y por supuesto de aquellas medianas y pequeñas que no están sujetas a la NFRD y la SFDR. De hecho, el pasado 21 de abril, la CE adoptó la propuesta Corporate Sustainability Reporting Directive (CSRD) (Comisión Europea, 2021a), que modificará la referida NFRD. La propuesta extiende el alcance de la obligatoriedad a todas las grandes empresas y a todas las empresas cotizadas en mercados regulados (excepto a las micro-empresas), requiere la auditoría (verificación) de la información reportada, introduce más requerimientos para un reporte detallado y conforme a los estándares obligatorios de la UE para el reporte de sostenibilidad, requiere a las empresas reportar la información en los formatos digitales adaptados para la lectura y registro en el Punto Europeo

\footnotetext{
${ }^{5}$ Para mayor información consultar en https://impactmanagementproject.com/

${ }^{6}$ Para mayor información consultar en www.iris.thegiin.org
} 
de Acceso único (ESAP) en el que la UE está trabajando en el marco de su Plan de Acción para la Unión de los Mercado de Capitales (Comisión Europea, 2020b). La CE ha realizado una consulta y pretende promulgar una Directiva para la creación del ESAP (Comisión Europea, 2021b) que centralice y homogenice la información financiera y no financiera publicada por las empresas. Habrá que asegurar la agilidad y la calidad de los modelos de reporte de información no-financiera en la pre-contratación de productos financieros e inversiones sostenibles, especialmente para el segmento minorista y PYMEs.

En resumen, dos retos son fundamentales. Primero, consensuar unas métricas de impacto y contribución alineadas con el logro de las metas del Acuerdo de París y los ODS. Segundo, acordar el cómo equilibrar el enfoque de la materialidad financiera asumido por el TFCD y por las ESAs con el enfoque de la materialidad social de las métricas referido en la NFRD y la Guía de la CE para el reporte de información no financiera sobre el clima. Y esto sería importante de cara a integrar la lógica de la dimensión social de las finanzas sostenibles dentro del marco normativo.

Asimismo, destacar la complejidad para su aplicación si atendemos al proceso aún en curso para la alineación de las RTS de las ESAs con la aplicación por niveles de la SFDR. El nivel 2 de la SFDR se aplicará en enero del 2022 y aún no se ha integrado dentro de las directivas sectoriales como las de Mercados de Instrumentos Financieros (MiFID), Pasaporte Europeo para las gestoras y fondos (UCITS y AIFMD), la de Distribución de Seguros (IDD), y las relativas a la supervisión en materia de solvencia, riesgos,..(Basilea, CRR/CRD,...).

Mencionar también que todavía no se habrían aclarado en la legislación los efectos en la responsabilidad de los administradores de las entidades sujetas a la NFRD y la SFDR respecto al cumplimiento diligente de estas nuevas obligaciones en materia de divulgación de información no financiera.

Por último, todavía muy pocas empresas estarían informando con suficientes indicadores del grado de progreso en la consecución de los ODS y Acuerdo de París por lo que sería importante generar nuevos indicadores sociales ligados a estos objetivos ya que el riesgo del Green y el Impact Washing es cada vez mayor por la movilización tan importante de recursos anunciada en el Plan de Inversión Sostenible de la UE. También sería de interés el estudiar la posibilidad de verificaciones independientes que aumenten la confianza de los inversores.

\subsubsection{CONSTRUCCIÓN DE ÍNDICES DE REFERENCIA O BENCHMARKS}

Sería necesario avanzar hacia una mayor transparencia sobre los detalles de la técnica de construcción, análisis, medición y agregación de los factores ASG de los índices de referencia de transición climática y/o sostenibilidad ya que sirven de referencia en las estrategias de inversión de cartera bajo criterios best in class o benchmark. El principal desafío radica en la diversidad y complejidad de las metodologías utilizadas por las CRA para la calificación de los activos, y en las evidencias de la baja correlación y alta dispersión de los resultados de las métricas ya comentadas en el apartado 3.1.4. La propia ESMA lo recoge en su ya citada carta de enero del 2021 a la CE advirtiendo que hay diferencias incluso en la construcción, agregación y ponderación de los indicadores y factores ASG.

Asimismo, las metodologías utilizadas para medir la huella de carbono, hídrica, medioambiental y/o social son complejas y no siempre coinciden. Las metodologías más avanzadas son las de medición de la huella de carbono, sin que por ello ninguna se haya convertido todavía en un referente universal (Foro Académico de Finanzas Sostenibles, 2020). La medición y gestión del impacto social (IMM) es incluso más compleja, 
coexistiendo también distintas metodologías (Trujillo et al., 2018). Tal y como señala el GIIN en su Encuesta Anual del 2020 (Global Impact Investing Network, 2020), los enfoques de IMM se están volviendo más estandarizados si bien la "fragmentación" permanecerá entre los desafíos para los próximos cinco años.

Cada índice en la práctica tiene una definición diferente de lo que significa un buen desempeño ASG y un sistema de ponderación diferente para los indicadores utilizados que pueden incluso no coincidir, ya que no existe todavía una batería estándar internacional de indicadores, y en muchos casos no siempre las propuestas de indicadores tienen el mismo enfoque o materialidad y responden a marcos diferentes (NFRD, TCFD, RTS de las ESAs,...).

Además, sin la necesaria transparencia en las metodologías y criterios de selección, una empresa situada en el primer nivel del índice podría ser la mejor o la "menos mala" de todas, y ello no implicaría necesariamente cumplir los objetivos perseguidos por el inversor. Disponer de metodologías claras, detalladas y normalizadas puede aportar gran valor para evitar ineficiencias en las estrategias ASG de los inversores. El Reglamento (UE) 2019/2089 obliga a los administradores, por ahora tan sólo de los índices climáticos, a publicar de qué manera se seleccionaron y ponderaron los activos subyacentes, y qué activos quedaron excluidos y por qué razón, cómo se han medido la huella de carbono y las emisiones de carbono de los activos, sus valores respectivos, incluida la huella de carbono total del índice de referencia, y el tipo y la fuente de los datos utilizados.

\subsection{GESTIÓN Y ANÁLISIS DE RIESGOS ASG}

Los retos en materia de integración y gestión de riesgos ASG son muchos y todavía inciertos. La EBA en el documento de consulta Gestión y Supervisión de Riesgos ASG referido anteriormente pone en evidencia algunos que resumimos a continuación:

- Perfeccionar la propia taxonomía para facilitar su aplicación y con ello favorecer un eficaz desarrollo de los sistemas de gestión y análisis de riesgos ASG (ARAS).

- Revisar la legislación de divulgación (NFRD y SFDR) para generar mayor alcance para un mayor número de empresas.

- Avanzar hacia una mayor estandarización de Métricas.

- Crear el ESAP con mediciones y métricas medioambientales por sectores.

- Avanzar en técnicas de análisis para establecer correlaciones entre riesgos ASG y riesgos financieros que sustente el necesario equilibrio entre la materialidad financiera y la materialidad social de la información no financiera y sus métricas.

- Incorporar análisis dinámicos de escenarios de más largo plazo acordes con la naturaleza y diversidad de los riesgos físicos y de transición climáticos.

- Analizar cómo lograr un equilibrio desde la óptica prudencial y de estabilidad financiera entre la supervisión de riesgos ASG y el impulso de la inversión sostenible en inversores cualificados con efecto arrastre como los Fondos de Pensiones. 
- Integrar los riesgos ASG dentro de la normativa sectorial relativas a la supervisión en materia de solvencia, riesgos,..(Basilea, CRR/CRD,...). En este sentido, en los prolegómenos de una posible incorporación de los riesgos climáticos de cara a la futura versión de Basilea, cabe mencionar que el Comité de Supervisión Bancaria de Basilea (BCBS), que preside el gobernador del Banco de España, Pablo Hernández de Cos, ha agregado el componente medioambiental entre sus prioridades de regulación y supervisión en el Programa de Estrategias y Prioridades para 2021 y 2022, que difundió el BCBS el pasado 16 de abril (Basel Committee on Banking Supervision, 2021). Entre ellas aparece "la valoración, la medición y mitigación de los riesgos financieros relacionados con el cambio climático" junto al impacto de la digitalización en el modelo de negocio y en las tasas de interés. No obstante, cuando se intentan medir estos riesgos surgen dificultades porque no se cuenta con la profundidad histórica suficiente y no existe experiencia previa con metodologías adaptadas para los cambios estructurales de esta magnitud que requieren una perspectiva a largo plazo. Tampoco se tienen análisis, lo que lleva a que las autoridades hayan optado por incluir el componente de cambio climático en las pruebas de resistencia de la EBA que se hagan a los bancos en 2022. En términos prácticos, el riesgo climático que incluye los riesgos físicos (catástrofes naturales) y de transición (transformación de las tecnologías y reasignación de actividades) podría recogerse en las categorías tradicionales de riesgos financieros (riesgos de crédito, de mercado, de liquidez o reputacional).

\subsection{INNOVACIÓN Y DESARROLLO DE PRODUCTOS DE FINANCIACIÓN E INVERSIÓN SOSTENIBLES}

A pesar del evidente grado de innovación expuesto en el apartado anterior, existen grandes desafíos tales como:

- Diseñar productos con un adecuado etiquetado y verificación dirigidos al segmento minorista.

- Profesionalizar y capacitar a todos los actores potenciales que puedan comercializar en el segmento minorista dichos productos.

- Incluir en el diseño de los productos los mecanismos de información pre-contractual y de seguimiento periódico que permitan una contratación diligente y una mayor disponibilidad de información no-financiera.

- Impulsar productos alineados con los ODS y con estrategias avanzadas de integración ASG y medición de Impacto.

- Fomentar productos de financiación combinada e instrumentos de colaboración público-privada para la inversión de impacto. Integrar la financiación adicional del sector público a la vez que se moviliza capital privado mediante mecanismos de asimetría en la asignación de los tramos de rentabilidad-riesgo según los distintos perfiles del inversor y de la inversión.

- Asegurar la transparencia y un reparto equilibrado de los costos de transacción en la contratación de los productos.

- Reconocer las diferencias regionales y de prioridades para un adecuado diseño de productos y una transición justa y equilibrada hacia una economía baja en carbono.

- Crear un marco fiscal, regulatorio y de supervisión que incentive el diseño y contratación de estos productos. 


\subsection{TECNOLOGÍAS DIGITALES}

Es también relevante destacar la complejidad del uso de tecnologías digitales para el desarrollo de las finanzas sostenibles. Por ejemplo, para el control y captura de datos en tiempo real de información climática, su análisis y tratamiento inteligente, la medición del impacto y su incorporación automática a los modelos de reporte y gestión de riesgos ASG para el debido cumplimiento de las obligaciones legislativas y de supervisión. Estas tecnologías pueden agilizar y hacer menos onerosa la divulgación y la posterior verificación de la información no financiera. La diversidad y complejidad de las tecnologías involucradas requiere de iniciativas sectoriales a modo de consorcio para profundizar en su testeo aplicado al desarrollo de las finanzas sostenibles.

Las tecnologías de análisis de datos e inteligencia artificial pueden aplicarse también a la construcción de escenarios futuros con horizontes temporales más largos, test de resistencia, y analizar con mayor rigor la materialidad financiera y social de los factores ASG analizados.

Por último, las tecnologías digitales pueden ser usadas para favorecer la inclusión financiera y la democratización de la financiación e inversión sostenible.

\section{CONCLUSIONES Y FUTURAS LÍNEAS DE INVESTIGACIÓN}

En el contexto actual del Covid-19 el sector bancario en Europa afronta múltiples dificultades vinculadas a las presiones en los márgenes por los bajos tipos de interés, requerimientos prudenciales, caída del volumen del negocio y calidad de los activos con el aumento consiguiente de provisiones. Ante ello, los supervisores han adoptado medidas para asegurar la estabilidad financiera en la UE que afectan a los requisitos de solvencia y a la asistencia de liquidez y garantías para que los bancos las canalicen a las empresas. Paralelamente se diseñó en la UE un Plan de Recuperación de la Economía (NextGeneration EU)’ que implica la movilización de financiación e inversiones para la transición hacia una economía y sociedad más sostenible, inclusiva, circular, y climáticamente neutra de aquí a 2050.

Para ello y según sostiene el Parlamento Europeo y Consejo de la UE en el citado Reglamento de la Taxonomía, "es necesario que las finanzas sostenibles estén plenamente integradas en el sistema financiero."

No sería desacertado afirmar que se han sentado las bases para un renovado contrato social que exigirá al sector bancario y financiero el cumplimiento de su compromiso con el desarrollo de las finanzas sostenibles. Su incumplimiento podría dar lugar a una pérdida de la confianza del consumidor en los productos y en la información que ofrecen las instituciones financieras, similar o mayor a la experimentada en ocasión de la crisis financiera del 2008 (Esteban-Sánchez et al, 2017).

Pero a pesar de los avances en la regulación, en la gestión de los riesgos ASG y en el desarrollo de productos de financiación e inversión sostenibles, existen importantes desafíos latentes para afrontar para el eficaz desarrollo de las finanzas sostenibles en Europa, entre los que destacan:

- Incrementar la disponibilidad, calidad y análisis de la información ASG alineada con los ODS y las metas del Acuerdo de París. Facilitar su reporte en la pre-contratación y seguimiento de los productos financieros y de inversión sostenibles.

- Crear una base de datos pública con métricas medioambientales por sectores y empresas, y promover la confluencia regulatoria y de recomendaciones sobre divulgación de información no financiera. 
- Fomentar el papel que pueden jugar las entidades financieras y proveedores de servicios ASG en el asesoramiento y capacitación a los segmentos de inversores minoristas y PYMEs.

- Estudiar un sistema de incentivos fiscales para el emisor/inversor que fomenten el desarrollo de un mercado de los productos financieros e inversiones sostenibles incluso a nivel minorista.

- Reducir los riesgos del green/impact washing mediante estandarización de métricas, criterios de sostenibilidad y etiquetado de productos con mayor verificación independiente.

- Avanzar en la integración de la regulación en la normativa sectorial (MIFID, UCITS, AIFMD, IDD, CRR/ CRD, Basilea,...).

- Avanzar en el desarrollo de la regulación relativa a la integración de la dimensión social y de gobernanza al actual marco normativo.

- Avanzar hacia enfoques de screening de inversiones basados en la integración estratégica ASG o el análisis del impacto positivo.

- Implementar ARAS y avanzar en la construcción de escenarios futuros y test de resistencia que permitan estudiar la materialidad financiera y social de los riesgos ASG dentro de un adecuado horizonte temporal.

- Analizar por los supervisores y reguladores las condiciones que deben cumplirse para lograr un equilibrio en la gestión de riesgos ASG desde la óptica prudencial de la supervisión de riesgos y estabilidad financiera.

- Coordinar programas de sensibilización y formación en finanzas sostenibles y de Inversión de Impacto dirigidas a todos los stakeholders implicados.

- Desarrollar Marcos nacionales y europeos de programas de Inversión de Impacto bajo esquemas de contratación y colaboración público-privada que fomente estrategias blended finance con mecanismos para el reparto y transferencias de riesgos, rentabilidades, responsabilidades de gestión y costes de transacción, y que logren movilizar capital privado y público.

- Promover el desarrollo de un sector de proveedores especializados de servicios ASG y de capacitación al tercer sector.

- Desarrollar Programas de Colaboración Internacional y tener en cuenta las diferencias regionales en las necesidades y prioridades para una transición justa y equilibrada.

- Reforzar las tecnologías digitales para el desarrollo de las finanzas sostenibles facilitando mayor transparencia, seguimiento en tiempo real, verificación del impacto y accesibilidad preservando los objetivos de inclusión y cohesión social.

Sería de interés abordar futuras líneas de investigación para armonizar métricas sobre cambio climático que propongan una aproximación cuantitativa para aquellos indicadores cualitativos sobre los que no existe consenso sobre su medición, y que puedan clasificarse bajo criterios de materialidad financiera y social. Todavía no se ha evaluado la diferencia que existe entre la información no financiera sobre cambio climático que publican las empresas y la que demandan los inversores. También cabría analizar métodos de construcción de escenarios con un enfoque largoplacista que permitan poner en relación riesgos financieros y riesgos ASG e incorporar las expectativas en las estimaciones de comportamiento de los distintos escenarios. 


\section{REFERENCIAS BIBLIOGRÁFICAS}

- Abrams, F. (1951). Management responsibilities in a complex world. Harvard Business Review, 29, 29-34.

- Banco Central Europeo (2020). Guía de referencia sobre riesgos relacionados con el clima y medioambientales. Disponible en https://www.bankingsupervision.europa.eu/ecb/pub/pdf/ssm.202011finalguideonclimaterelatedandenvironmentalrisks 58213f6564.es.pdf

- Basel Committee on Banking Supervision (2021). Basel Committee work programme and strategic priorities for 2021/22. Bank for International Settlements. https://www.bis.org/bcbs/bcbs_work.pdf

- Berg et al. (2019). Aggregate confusion: The divergence in ESG ratings, MIT Sloan SchoolWorking Paper, (5822-19), 1-42. Disponible en https://www.eticanews.it/wp-content/uploads/2019/09/20190819MITd ivergenceESGratings.pdf

- BlackRock Financial Markets Advisory (2020). Interim study on the development of tools and mechanisms for the integration of environmental, social and governance (ESG) factors into the EU banking prudential framework and into banks' business strategies and investment policies. Disponible en https://ec.europa. eu/info/publications/201214-interim-study-esg-factors-banking_en

- Bolton, P., Després, M., Pereira Da Silva, L., Samama, F. y Svartzman, R. (2020). The green swan. Bank for International Settlements, January.

- Bowen HR. (1953). Social Responsibilities of the Businessman. New York: Harper and Row.

- Carbon Disclosure Project (CDP); Climate Disclosure Standards Board (CDSB); GRI; International Integrated Reporting Council (IIRC) y Sustainability Accounting Standards Board (SASB) (2020). Reporting on enterprise value Illustrated with a prototype climate-related financial disclosure standard. Impact Management Project, World Economic Forum and Deloitte. Disponible en https://incp.org.co/ Site/publicaciones/info/archivos/Reporting-on-enterprise-value-05012021.pdf

- Carroll, A.B. (1979). A three-dimensional conceptual model of corporate performance. Academy of Management Journal, 4(4), 497-505. DOI: 10.2307/257850

- Carroll A.B. (1981). Business and society: managing corporate social performance. Boston: Little Brown.

- Carroll A.B. (1991). The pyramid of corporate social responsibility: Toward the moral management of organizational stakeholders. Business Horizons, 34(4), 39-48. DOI: 10.1016/0007-6813(91)90005-G

- Carroll A.B. (1999). Corporate social responsibility. Evolution of a definitional construction. Business and Society 38(3), 268-295. DOI: 10.1177/000765039903800303

- Comisión Europea (2017). Comunicación C215/1 Directrices sobre la presentación de informes no financieros. Disponible en https://eur-lex.europa.eu/legal-content/ES/TXT/PDF/?uri=CELEX:52017XC0 705(01)\&from=EN

» (2018). Comunicación 97 final, Plan de Acción: Financiar el desarrollo sostenible, Disponible en https:// eur-lex.europa.eu/legal-content/ES/TXT/PDF/?uri=CELEX:52018DC0097\&from=EN

» (2019a). Comunicación 640 final, El Pacto Verde Europeo. Disponible en https://eur-lex.europa.eu/resource.html?uri=cellar:b828d165-1c22-11ea-8c1f-01aa75ed71a1.0004.02/DOC_1\&format=PDF 
" (2019b). Guidelines on Reporting Climate-Related Information. Disponible en https://ec.europa.eu/finance/docs/policy/190618-climate-related-information-reporting-guidelines_en.pdf

" (2020a). Comunicación 21 final, Plan de Inversiones para una Europa Sostenible. Disponible en https:// eur-lex.europa.eu/legal-content/ES/TXT/PDF/?uri=CELEX:52020DC0021\&from=EN

" (2020b). Anexo de la Comunicación, Una Unión de los Mercados Capitales para las personas y las empresas: nuevo plan de acción. Disponible en https://eur-lex.europa.eu/resource.html?uri=cellar:61042990fe46-11ea-b44f-01aa75ed71a1.0005.02/DOC_2\&format=PDF

" (2021a). Propuesta de Directiva del Parlamento Europeo y del Consejo por la que se modifican la Directiva 2013/34/UE, la Directiva 2004/109/CE, la Directiva 2006/43/CE y el Reglamento (UE) n. ${ }^{\circ}$ $537 / 2014$, por lo que respecta a la información corporativa en materia de sostenibilidad. Disponible en https://eur-lex.europa.eu/legal-content/ES/TXT/PDF/?uri=CELEX:52021PC0189\&from=EN

" (2021b). Targeted Consultation Document, Establishment of a European Single Access Point (ESAP) for Financial and Non-financial information publicly disclosed by companies. Disponible en https:// ec.europa.eu/info/sites/default/files/business_economy_euro/banking_and_finance/documents/2021european-single-access-point-consultation-document_en.pdf

- Emshoff, J. R. y Freeman, R. E. (1978). Stakeholder Management, The Wharton Applied Research Center, Working paper (July), 3-78.

- Esteban-Sánchez, P., De la Cuesta-González, M. y Paredes-Gázquez, J.D. (2017). Corporate social performance and its relation with corporate financial performance: International evidence in the banking industry, Journal of Cleaner Production, 162(20), 1102-1110. DOI: 10.1016/j.jclepro.2017.06.127

- Evan, W.M. y Freeman, R.E. (1988). A Stakeholder Theory of the modern corporation: Kantian capitalism. En T. Beauchamp y N. Bowie (ed.): Ethical Theory Business. Prentice Hall. Englewood Cliffs, 75-93.

- European Banking Authority (2020), EBA Discussion paper. On management and supervision of ESG risks for credit institutions and investment firms. Disponible en https://www.eba.europa.eu/sites/default/ documents/files/document_library/Publications/Discussions/2021/Discussion\%20Paper\%20on\%20 management\%20and\%20supervision\%20of\%20ESG\%20risks\%20for\%20credit\%20institutions\%20 and\%20investment\%20firms/935496/2020-11-02\%20\%20ESG\%20Discussion\%2OPaper.pdf

- European Insurance and Occupational Pensions Authority (2021), European Final Report on draft Regulatory Technical Standards. Disponible en https://www.eiopa.europa.eu/content/final-report-draftregulatory-technical-standards_en

" (2021). Carta de la ESMA a la CE. https://www.esma.europa.eu/sites/default/files/library/esma30-379423_esma_letter_to_ec_on_esg_ratings.pdf

- European Securities and Markets Authority (2019). Final Report: Guidelines on Disclosure Requirements Applicable to Credit Ratings. Disponible en https://www.esma.europa.eu/sites/default/files/library/ esma33-9-320_final_report_guidelines_on_disclosure_requirements_applicable_to_credit_rating_ agencies.pdf

- EU High-Level Expert Group on Sustainable Finance (2018). Final report 2018. Disponible en https:// ec.europa.eu/info/sites/default/files/180131-sustainable-finance-final-report_en.pdf 
- EU Technical Expert Group on Sustainable Finance (2019). Report on EU Green Bond Standard. Disponible en https://ec.europa.eu/info/sites/info/files/business_economy_euro/banking_and_finance/ documents/190618-sustainable-finance-teg-report-green-bond-standard_en.pdf

- Foro Académico de Finanzas Sostenibles (2020). Metodologías de análisis para el alineamiento de carteras financieras con la acción climática. https://s3-eu-west-1.amazonaws.com/landingi-editor-uploads/ Z1xF2844/Monografia002_Alineamiento_de_carteras_financieras_final.pdf

- Fondo Mundial para la Naturaleza (2020). "Estrategias de engagement del sector financiero para acelerar la descarbonización”. Disponible en https://wwfes.awsassets.panda.org/downloads/estrategias_de_ engagement_del_sector_finaciero_para_acelerar_la_descarbonizacion.pdf

- Freeman, R.E. (1984). Strategic Management: a Stakeholder Approach. Boston, Massachusetts, EE.UU: Pitman Publishing.

- Freeman, R.E. (1994). The Politics of Stakeholder Theory: Some future directions. Business Ethics Quarterly, 4(4), 409-429. DOI: 10.2307/3857340

- Global Impact Investing Network (2020). Annual Impact Investor Survey. Executive summary. Disponible en https://thegiin.org/assets/GIIN\%20Annual\%2OImpact\%2OInvestor\%20Survey\%202020\%20Executive\%20 Summary.pdf

- Global Sustainable Investment Alliance (2018). Global Sustainable Investment Review, (2018). Disponible en http://www.gsi-alliance.org/trends-report-2018/ (consulta el 25 de febrero de 2021).

- International Platform on Sustainable Finance (2020). Annual Report, October. Disponible en https://ec.europa.eu/info/sites/info/files/business_economy_euro/banking_and_finance/documents/ international-platform-sustainable-finance-annual-report-2020_en.pdf

- Joint Committee of the European Supervisory Authorities (2021). European Final Report on draft Regulatory Technical Standards. Disponible en https://www.eiopa.europa.eu/content/final-report-draftregulatory-technical-standards_en

- Naciones Unidas (1999). Pacto Mundial de las Naciones Unidas Global Compact. Disponible en https:// www.unglobalcompact.org/what-is-gc/mission/principles

» (2008). International Standard Industrial Classification of All Economic Activities (ISIC), Rev. 4. Disponible en https://unstats.un.org/unsd/publication/seriesm/seriesm_4rev4e.pdf

- Observatorio Español de la Financiación Sostenible (2020). Informe Anual. Disponible en https://ofiso.es/ files/Informe-Anual-OFISO-2020.pdf

- Parlamento Europeo y Consejo de la Unión Europea (2014). Directiva 2014/95/UE por la que se modifica la Directiva 2013/34/UE en lo que respecta a la divulgación de información no financiera e información sobre diversidad por parte de determinadas grandes empresas y determinados grupos. Disponible en https://eur-lex.europa.eu/legal-content/ES/TXT/PDF/?uri=CELEX:32014L0095\&from=EN

" (2017). Directiva UE 2017/828 por la que se modifica la Directiva 2007/36/CE en lo que respecta al fomento de la implicación a largo plazo de los accionistas. Disponible en https://eur-lex.europa.eu/legalcontent/ES/TXT/PDF/?uri=CELEX:32017L0828\&from=ES 
" (2019a). Reglamento (UE) 2019/2088 sobre divulgación de información sobre sostenibilidad en el sector financiero. Disponible en https://eur-lex.europa.eu/legal-content/ES/TXT/PDF/?uri=CELEX:32019 R2088\&from $=E S$

» (2019b). Reglamento (UE) 2019/2089 por el que se modifica el Reglamento (UE) 2016/1011 en lo relativo a los índices de referencia de transición climática de la UE, los índices de referencia de la UE armonizados con el Acuerdo de París y la divulgación de información relativa a la sostenibilidad de los índices de referencia. Disponible en https://eur-lex.europa.eu/legal-content/ES/TXT/PDF/?uri=CELEX:32019 R2089\&from $=E S$

" (2019c). Reglamento (UE) n 2019/876 por el que se modifica el Reglamento (UE) 575/2013 sobre los requisitos prudenciales de las entidades de crédito y las empresas de inversión. Disponible en https:// eur-lex.europa.eu/legal-content/ES/TXT/PDF/?uri=CELEX:32019R0876\&from=EN

" (2019d). Directiva 2019/878 que modifica la Directiva 2013/36/UE relativa al acceso a la actividad de las entidades de crédito y a la supervisión prudencial de las entidades de crédito y las empresas de inversión. Disponible en https://eur-lex.europa.eu/legal-content/EN/TXT/PDF/?uri=CELEX:32019L08 $78 \&$ from $=\mathrm{EN}$

" (2020). Reglamento UE 2020/852 relativo al establecimiento de un marco para facilitar las inversiones sostenibles y por el que se modifica el Reglamento (UE) 2019/2088. Disponible en https://eur-lex.europa.eu/legal-content/ES/TXT/PDF/?uri=CELEX:32020R0852\&from=EN

- Task Force on Climate-related Financial Disclosures (2017). Recommendations of the Task Force on Climate-related Financial Disclosures. Disponibleen https://assets.bbhub.io/company/sites/60/2020/10/ FINAL-2017-TCFD-Report-11052018.pdf

- Trujillo, R.; Gómez, A. y Canales, R. (2018). Midiendo el valor del Impacto Social Empresarial. FORÉTICA". https://foretica.org/wp-content/uploads/2018/12/midiendo_el_valor_del_impacto_social_ empresarial-1.pdf

- UniónEuropea(2010). Diario Oficial delaUE.Versiónconsolidada del Tratado Oficial de la UE Disponibleen https://eur-lex.europa.eu/resource.html?uri=cellar:2bf140bf-a3f8-4ab2-b506-fd71826e6da6.0005.02/ DOC_1\&format=PDF

- UNEP Finance Initiative (UNEPFI) y European Banking Federation (EBF) (2021). Testing the application of the EU Taxonomy to core banking products: High level recommendations. Disponible en https://www. ebf.eu/wp-content/uploads/2021/01/Testing-the-application-of-the-EU-Taxonomy-to-core-bankingproducts-EBF-UNEPFI-report-January-2021.pdf

- Wood, D. J. y Lodgson J.M. (2002). Business Citizenship: From Individuals to Organizations. Business Ethics Quarterly, Ruffin Series, 3, 59-94. DOI: 10.5840/ruffinx200232

- World Commission on Environment and Development (1987). Our Common Future. Oxford: Oxford University Press. 
Tabla 1. Lista de iniciativas de finanzas sostenibles

\begin{tabular}{|c|c|c|c|c|c|c|c|}
\hline 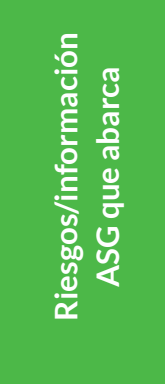 & 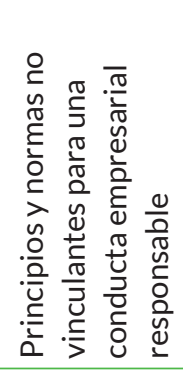 & 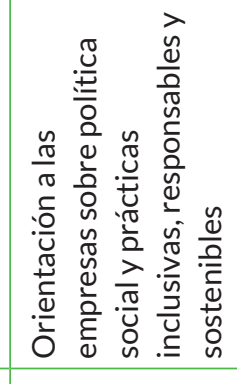 & 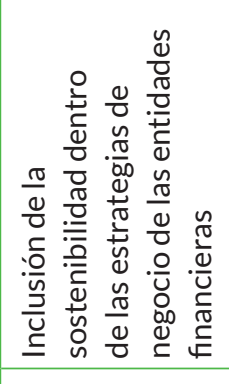 & 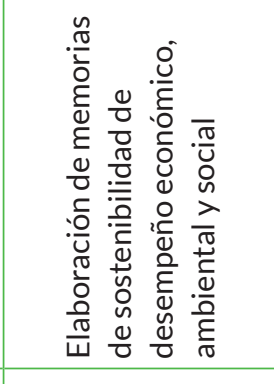 & 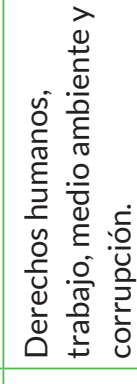 & 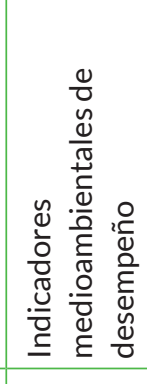 & 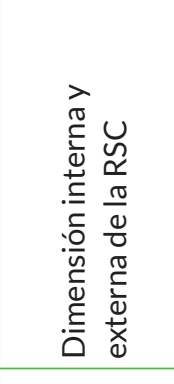 \\
\hline 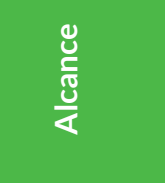 & $\begin{array}{l}\overline{\widetilde{J}} \\
\overline{0} \\
\mathrm{O}\end{array}$ & $\begin{array}{l}\bar{\Omega} \\
\overline{0} \\
\overline{0}\end{array}$ & $\begin{array}{l}\bar{\Omega} \\
\overline{0} \\
\overline{0}\end{array}$ & $\begin{array}{l}\bar{\Omega} \\
\overline{0} \\
\overline{0}\end{array}$ & $\begin{array}{l}\bar{\sigma} \\
\overline{0} \\
\overline{0}\end{array}$ & 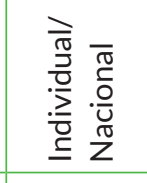 & 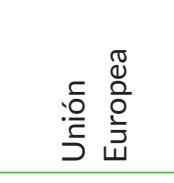 \\
\hline 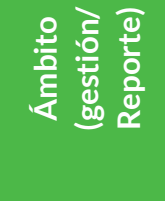 & 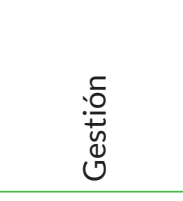 & 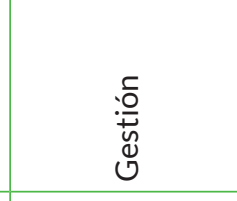 & 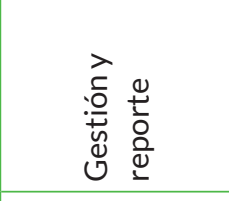 & 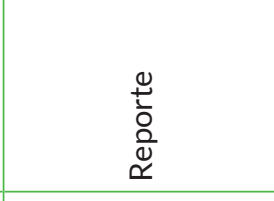 & 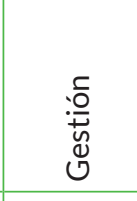 & 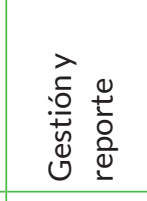 & 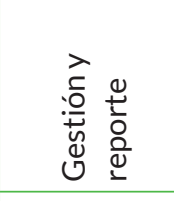 \\
\hline 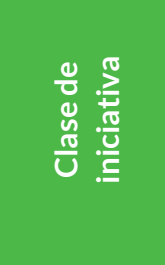 & 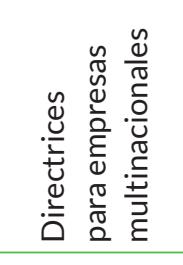 & 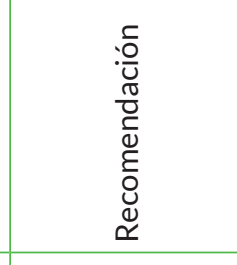 & 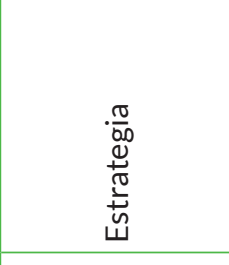 & 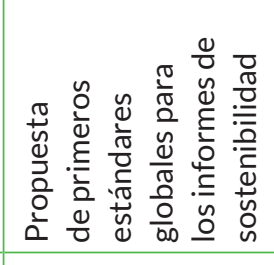 & 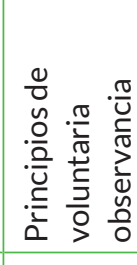 & 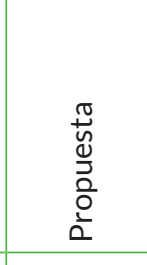 & 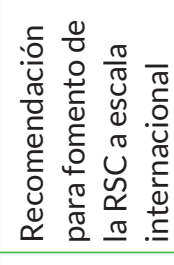 \\
\hline 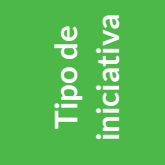 & 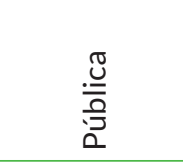 & 兽 & 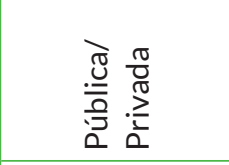 & 预 $\frac{\pi}{0}$ & 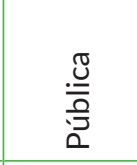 & $\begin{array}{l}\frac{\pi}{\pi} \\
\stackrel{\pi}{2} \\
\stackrel{2}{0}\end{array}$ & 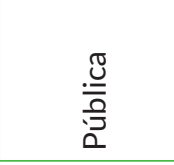 \\
\hline 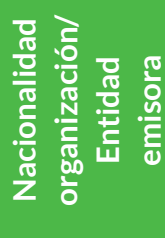 & 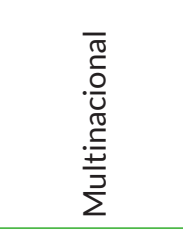 & 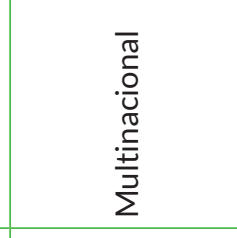 & 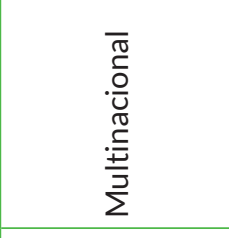 & 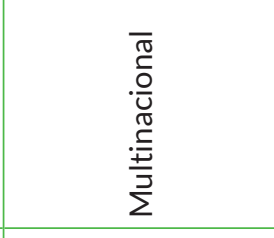 & 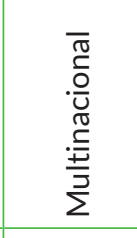 & & 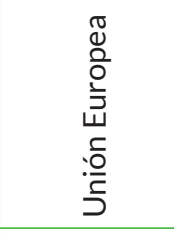 \\
\hline 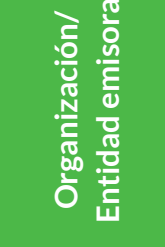 & U్ & 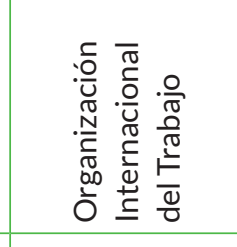 & 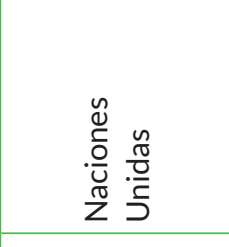 & 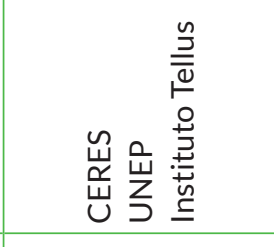 & 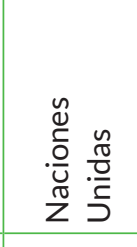 & 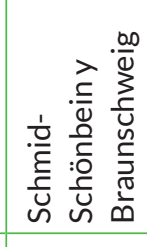 & 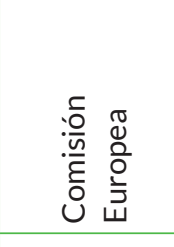 \\
\hline 察誉言 & $\stackrel{0}{\stackrel{2}{\sigma}}$ & $\underset{\sigma}{\hat{\sigma}}$ & $\begin{array}{l}\alpha \\
\text { - }\end{array}$ & $\begin{array}{l}\alpha \\
\stackrel{\sigma}{\sigma}\end{array}$ & ষ্ণ & ষ্ণ & ర్రి \\
\hline 竞 & 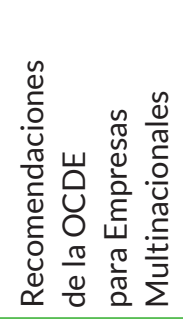 & 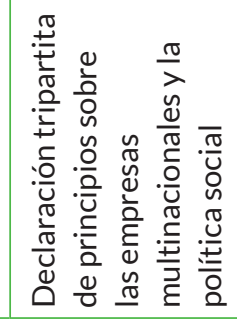 & 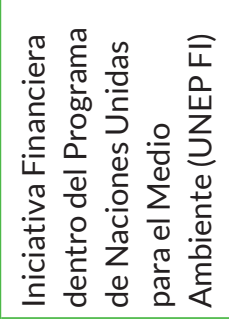 & 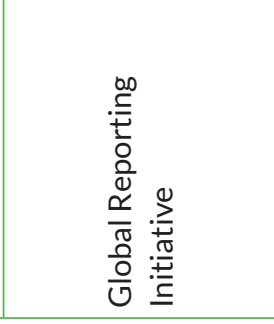 & 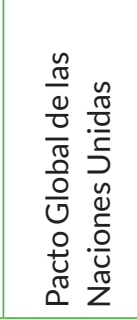 & 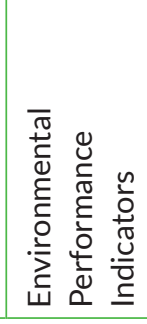 & 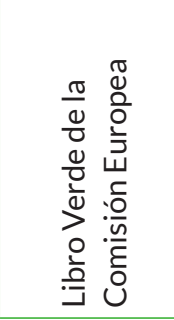 \\
\hline
\end{tabular}




\begin{tabular}{|c|c|c|c|c|c|c|c|}
\hline 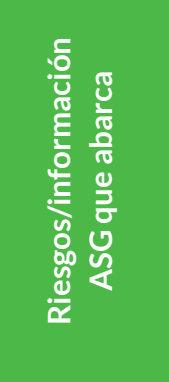 & 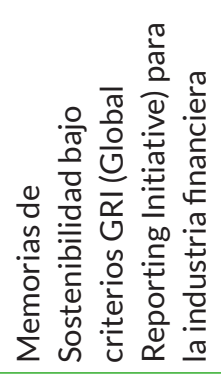 & 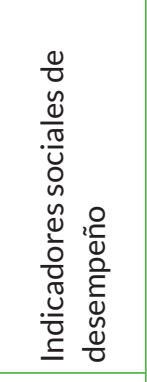 & 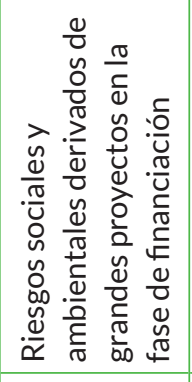 & 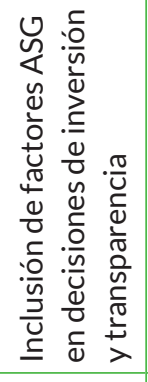 & 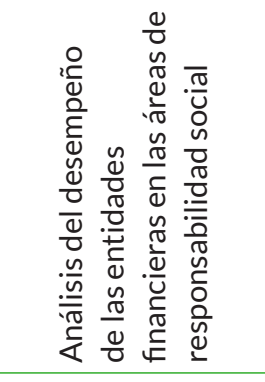 & 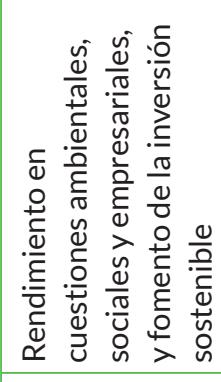 & 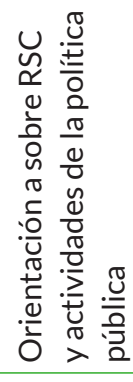 \\
\hline $\begin{array}{l}\frac{\mathbb{J}}{\frac{\mathrm{E}}{\mathrm{J}}} \\
\frac{\mathrm{J}}{4}\end{array}$ & $\begin{array}{l}\bar{\pi} \\
\frac{0}{0} \\
0\end{array}$ & 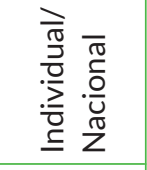 & $\begin{array}{l}\bar{\pi} \\
\frac{\pi}{0} \\
\frac{0}{U}\end{array}$ & $\begin{array}{l}\bar{\pi} \\
\frac{0}{0} \\
\frac{0}{U}\end{array}$ & $\begin{array}{l}\bar{\pi} \\
\frac{0}{0} \\
\frac{0}{U}\end{array}$ & $\begin{array}{l}\bar{\pi} \\
\frac{0}{0} \\
\frac{0}{v}\end{array}$ & $\begin{array}{l}\bar{\pi} \\
\stackrel{0}{0} \\
\end{array}$ \\
\hline 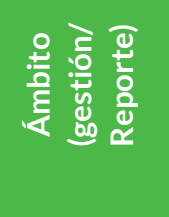 & 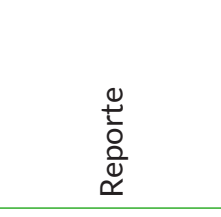 & 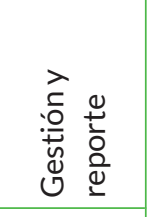 & & 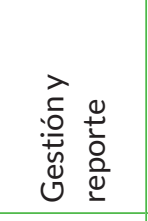 & 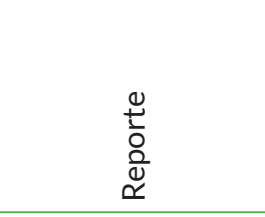 & 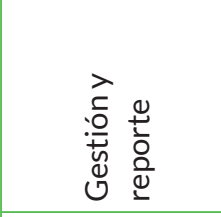 & 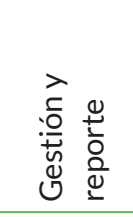 \\
\hline 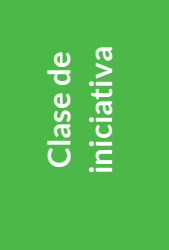 & $\begin{array}{l}\frac{\pi}{40} \\
\frac{0}{0} \\
\frac{0}{0} \\
0\end{array}$ & 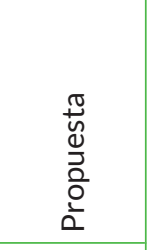 & 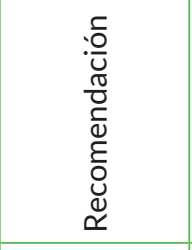 & 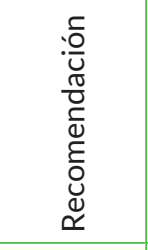 & $\begin{array}{l}\frac{\pi}{0} \\
\stackrel{0}{0} \\
\frac{0}{0} \\
\frac{0}{0}\end{array}$ & 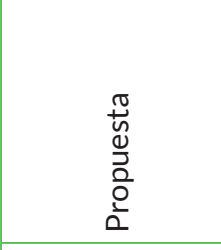 & 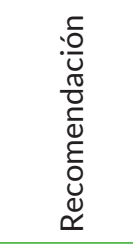 \\
\hline 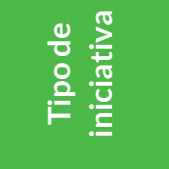 & 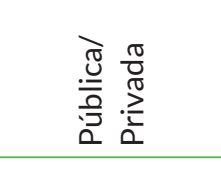 & 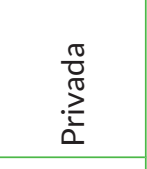 & 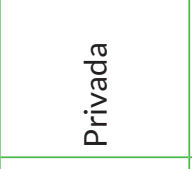 & $\frac{\frac{\pi}{\bar{\sigma}}}{\frac{\bar{z}}{2}}$ & 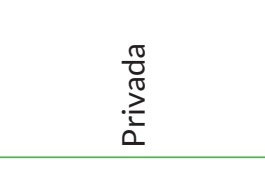 & 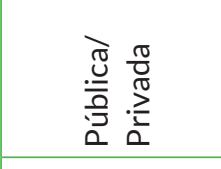 & 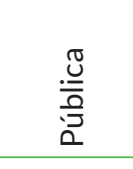 \\
\hline 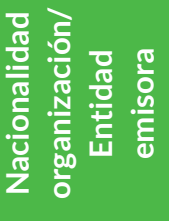 & 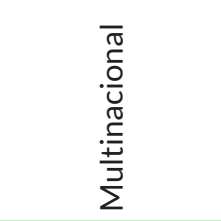 & 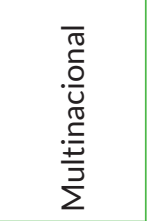 & 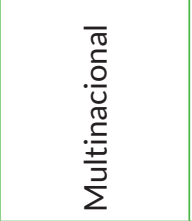 & 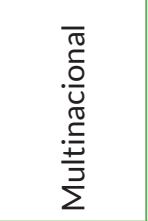 & 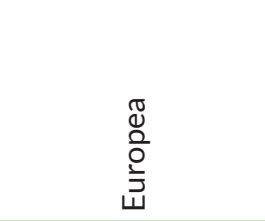 & 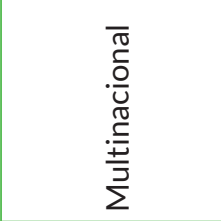 & 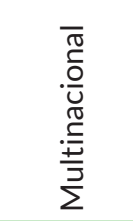 \\
\hline 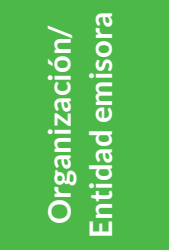 & 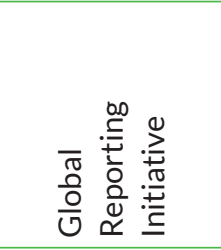 & 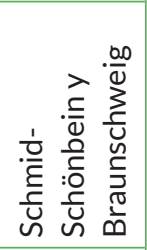 & 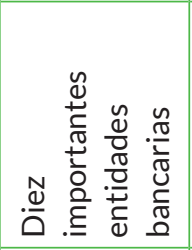 & 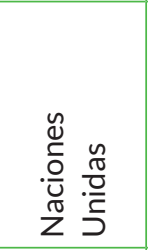 & $\frac{\varrho}{\frac{\varrho}{w}}$ & 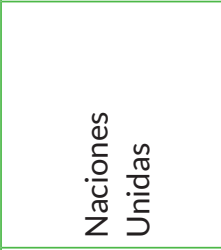 & 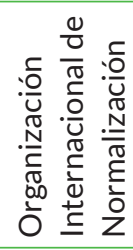 \\
\hline 选造 & ণิ & ণิ & రి & ঠ্ণ & ¿্ণ & ¿̊̀ & 옴 \\
\hline & 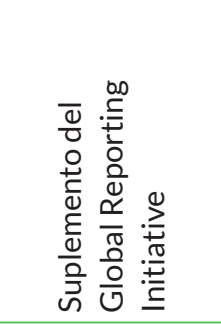 & 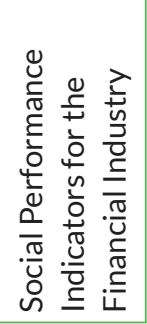 & 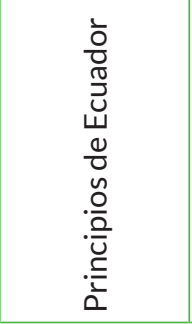 & 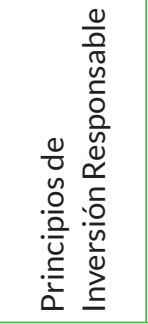 & 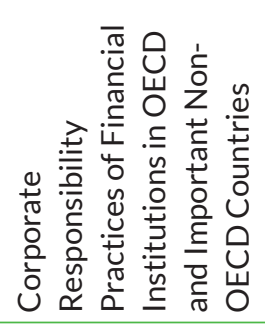 & 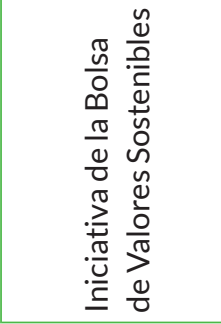 & 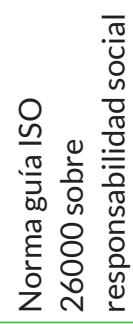 \\
\hline
\end{tabular}




\begin{tabular}{|c|c|c|c|c|c|c|c|}
\hline 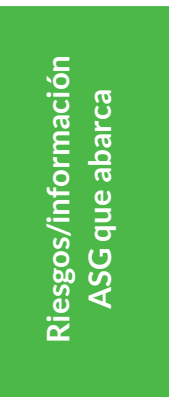 & 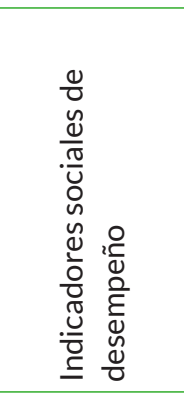 & 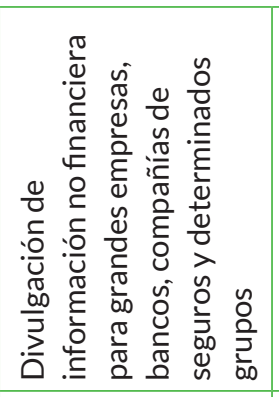 & 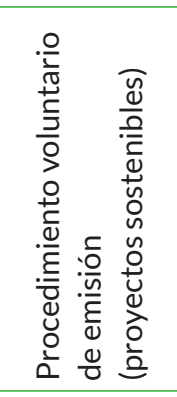 & 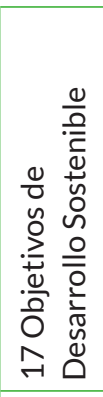 & 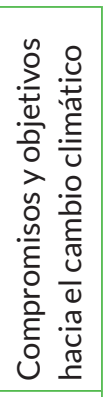 & 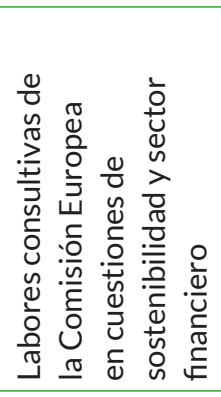 & 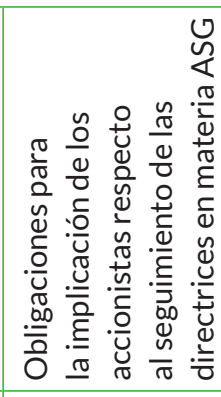 \\
\hline 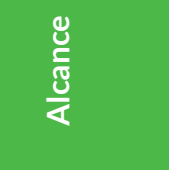 & $\begin{array}{l}\bar{\pi} \\
\frac{0}{0} \\
U\end{array}$ & 这 & $\begin{array}{l}\bar{\pi} \\
\frac{0}{0} \\
\frac{0}{v}\end{array}$ & $\begin{array}{l}\bar{\pi} \\
\frac{0}{0} \\
\frac{0}{v}\end{array}$ & $\begin{array}{l}\bar{\pi} \\
\frac{0}{0} \\
\end{array}$ & $\begin{array}{l}\bar{\pi} \\
\frac{0}{0} \\
v\end{array}$ & 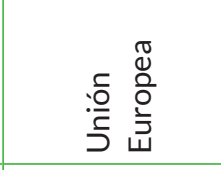 \\
\hline 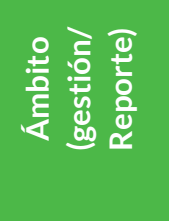 & 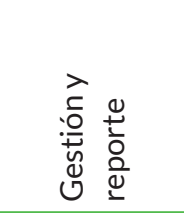 & 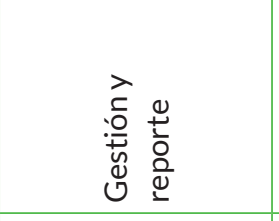 & 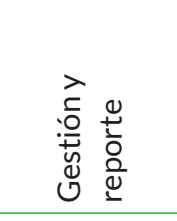 & 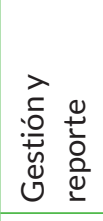 & 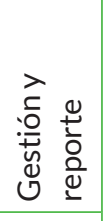 & 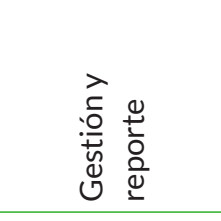 & 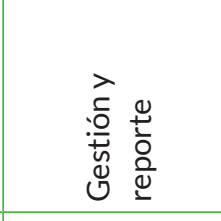 \\
\hline 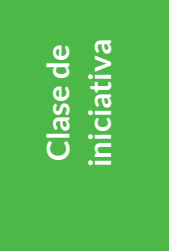 & $\begin{array}{l}\frac{\pi}{U} \\
0 \\
0 \\
0 \\
0 \\
0\end{array}$ & 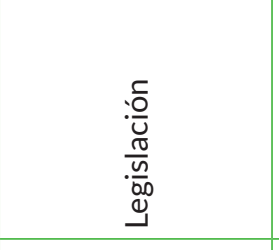 & 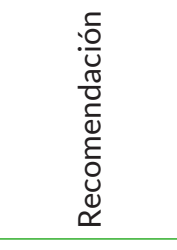 & 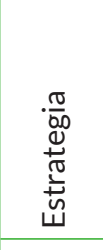 & 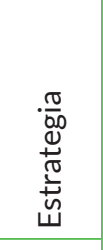 & 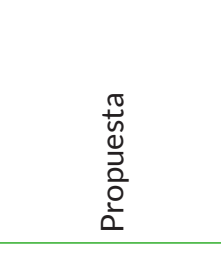 & 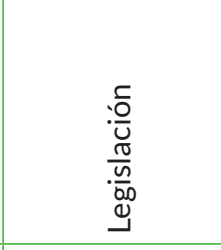 \\
\hline 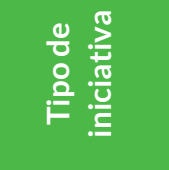 & 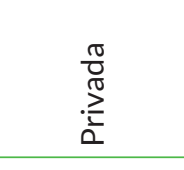 & $\frac{\sqrt[\pi]{0}}{\frac{0}{2}}$ & 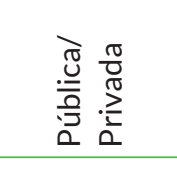 & $\frac{\sqrt[0]{0}}{\frac{0}{3}}$ & $\frac{\sqrt[0]{0}}{\frac{0}{0}}$ & 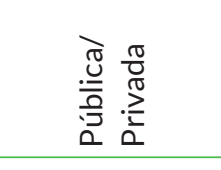 & $\frac{\frac{\pi}{0}}{\frac{0}{2}}$ \\
\hline 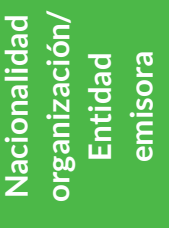 & 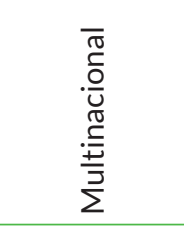 & 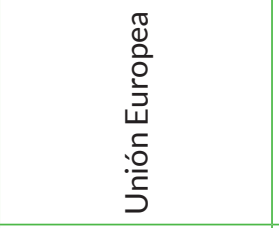 & 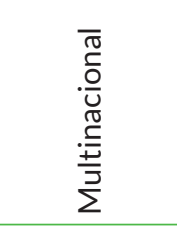 & 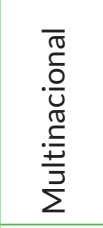 & 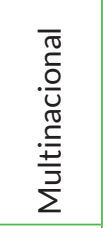 & 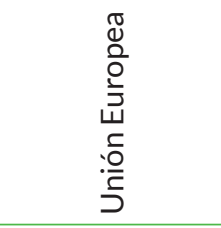 & 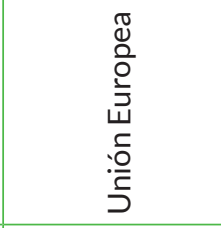 \\
\hline 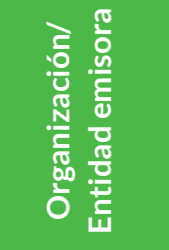 & 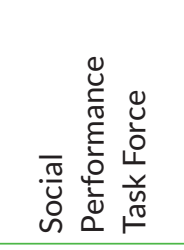 & 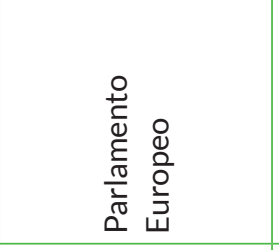 & 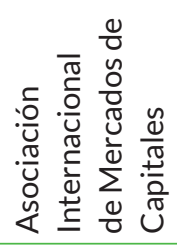 & 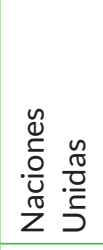 & 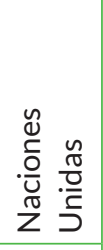 & 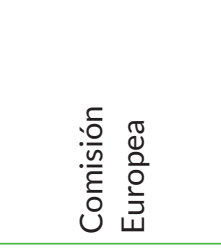 & 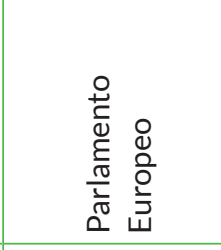 \\
\hline 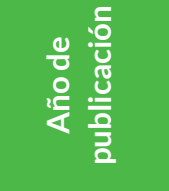 & خิ & $\stackrel{\sim}{\stackrel{\sim}{\sim}}$ & $\stackrel{\sim}{\stackrel{\sim}{2}}$ & $\stackrel{n}{\stackrel{n}{2}}$ & $\stackrel{n}{\stackrel{n}{2}}$ & $\stackrel{\circ}{\circ}$ & ิે̀) \\
\hline 总 & 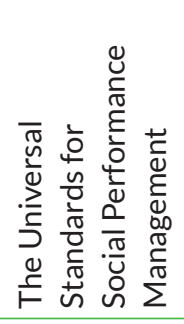 & 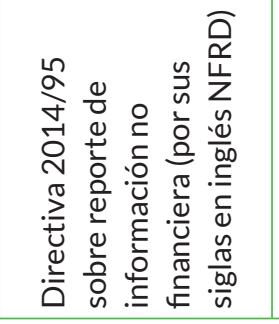 & 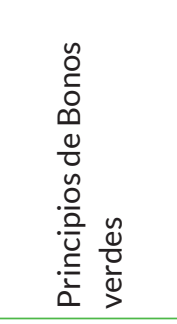 & 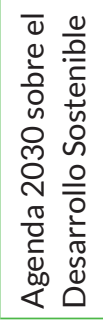 & 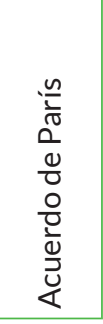 & 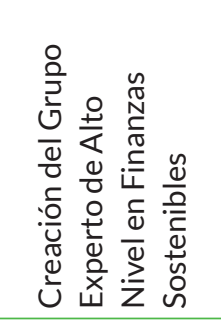 & 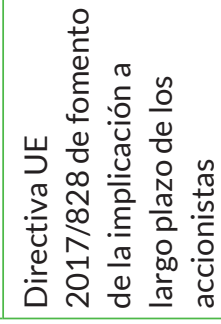 \\
\hline
\end{tabular}




\begin{tabular}{|c|c|c|c|c|c|c|}
\hline 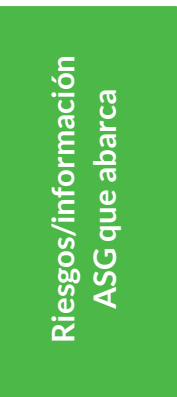 & 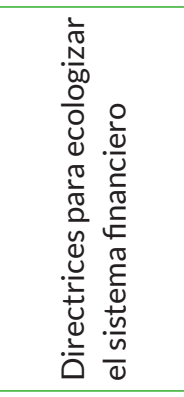 & 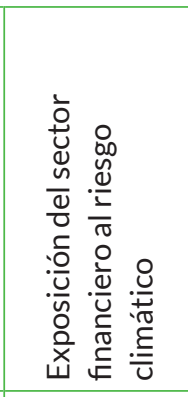 & 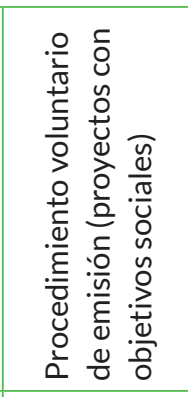 & 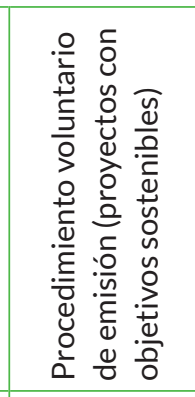 & 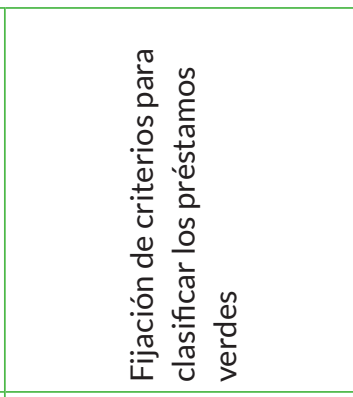 & 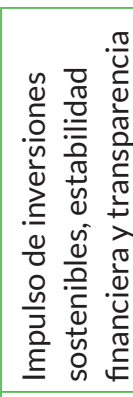 \\
\hline 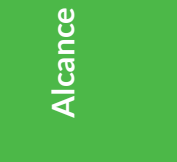 & $\begin{array}{l}\bar{\pi} \\
\stackrel{0}{0} \\
\end{array}$ & $\begin{array}{l}\overline{0} \\
\text { 은 } \\
\end{array}$ & $\begin{array}{l}\overline{0} \\
\text { 음 } \\
\text { U. }\end{array}$ & $\begin{array}{l}\bar{\pi} \\
\frac{0}{0} \\
\frac{0}{U}\end{array}$ & $\begin{array}{l}\overline{0} \\
\stackrel{0}{0} \\
\end{array}$ & $\begin{array}{l}\overline{\widetilde{\sigma}} \\
\frac{\mathrm{O}}{\mathrm{U}}\end{array}$ \\
\hline 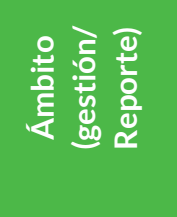 & 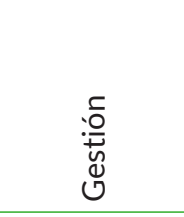 & 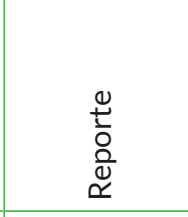 & 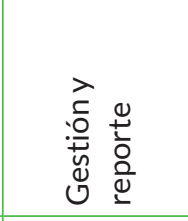 & 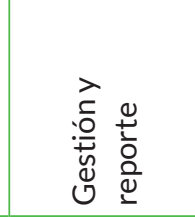 & :음 & 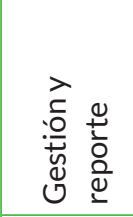 \\
\hline 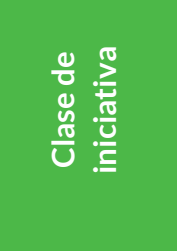 & 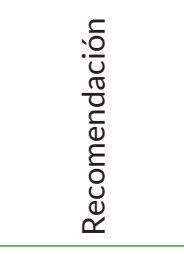 & 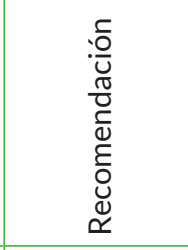 & 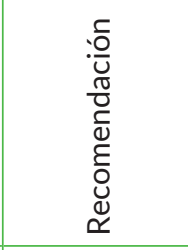 & 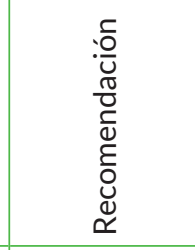 & 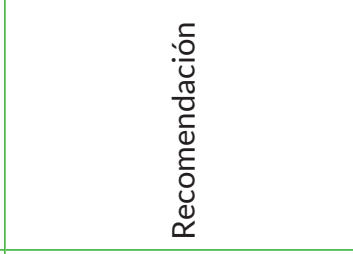 & 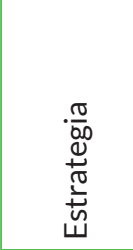 \\
\hline 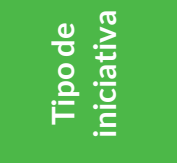 & $\frac{.00}{\frac{\pi}{3}}$ & 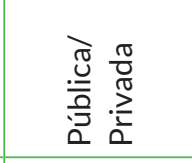 & 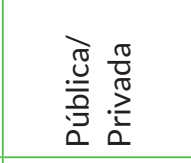 & 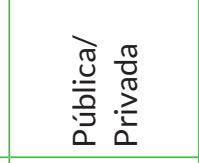 & 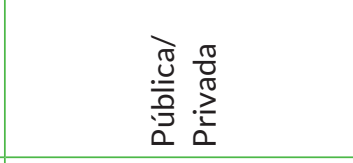 & $\frac{.00}{\frac{0}{3}}$ \\
\hline 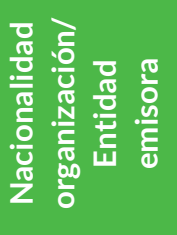 & 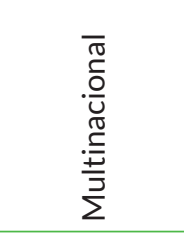 & 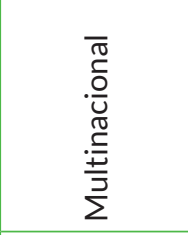 & 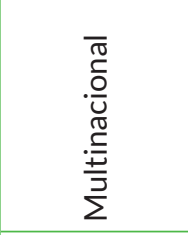 & 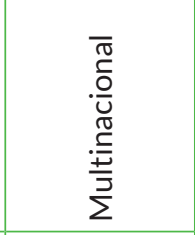 & 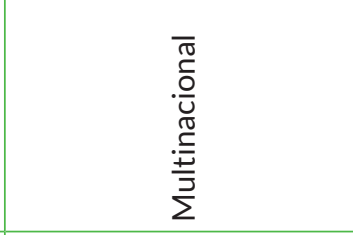 & 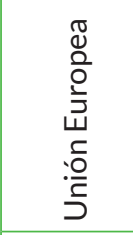 \\
\hline 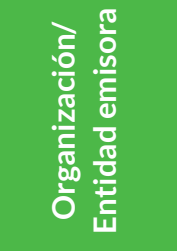 & 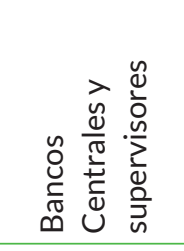 & 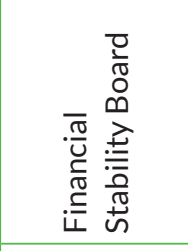 & 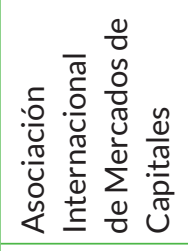 & 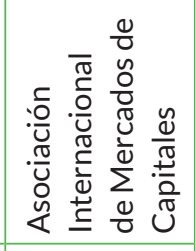 & 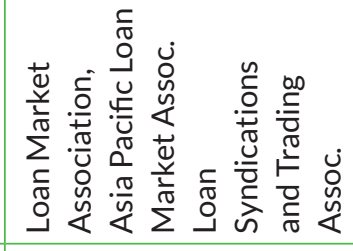 & 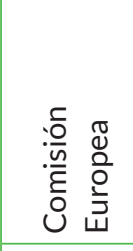 \\
\hline 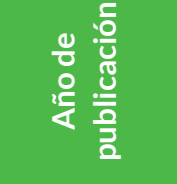 & 㝏 & 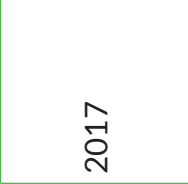 & ને & 今ે & $\stackrel{\infty}{\stackrel{1}{N}}$ & $\stackrel{\infty}{\vec{N}}$ \\
\hline 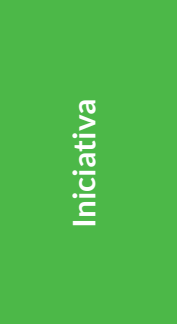 & 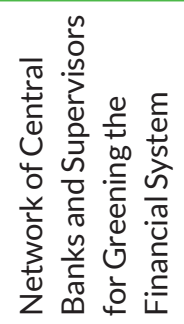 & 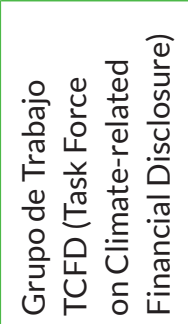 & 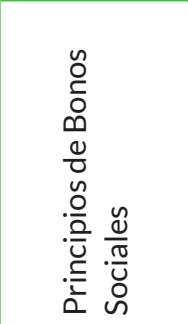 & 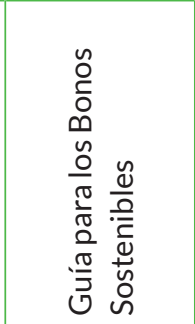 & 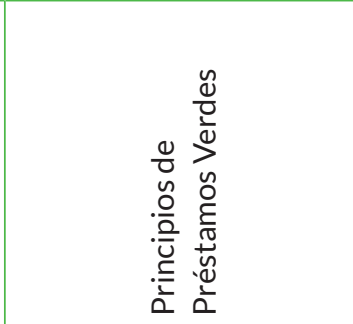 & 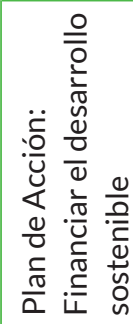 \\
\hline
\end{tabular}




\begin{tabular}{|c|c|c|c|c|c|c|}
\hline 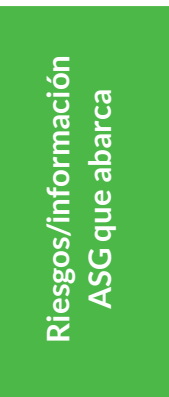 & 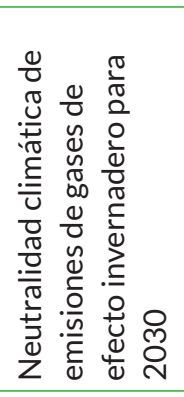 & 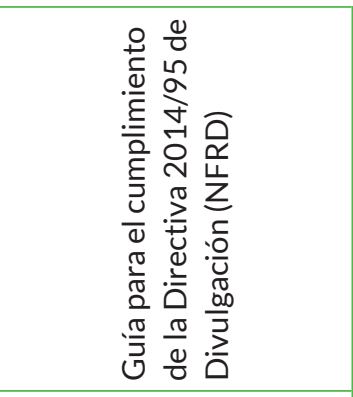 & 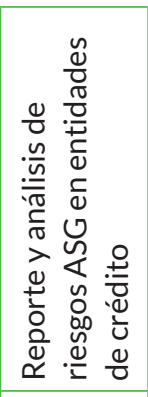 & 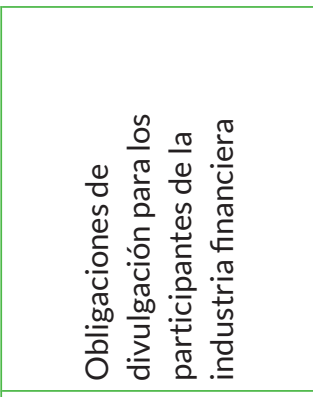 & 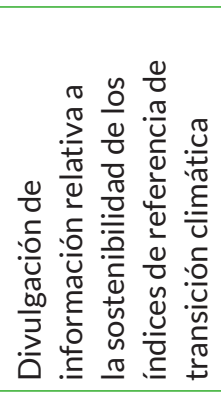 & 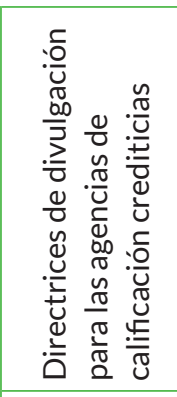 \\
\hline 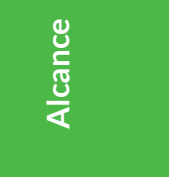 & شَ & 离 & 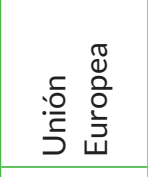 & لَّ & 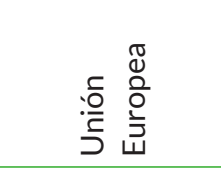 & لَّ \\
\hline 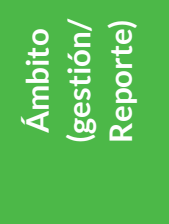 & 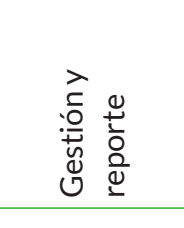 & 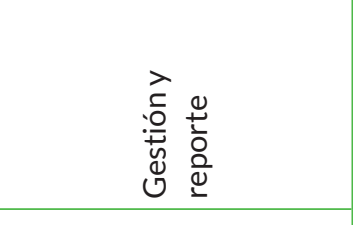 & 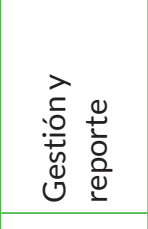 & 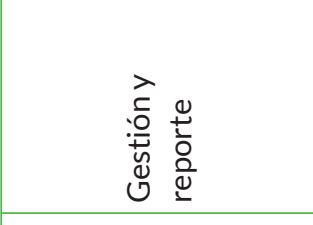 & 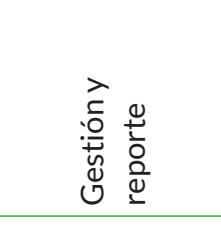 & 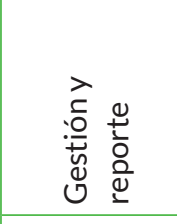 \\
\hline 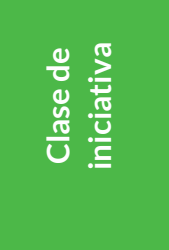 & 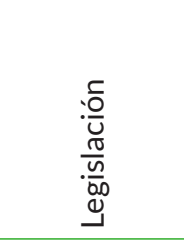 & 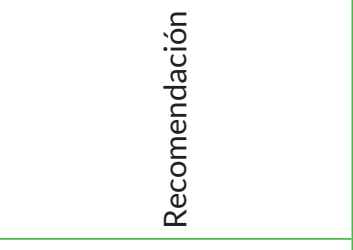 & 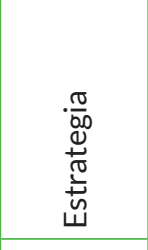 & 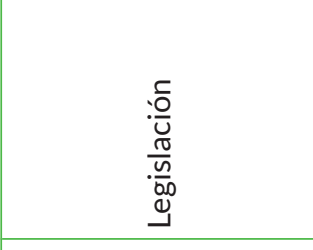 & 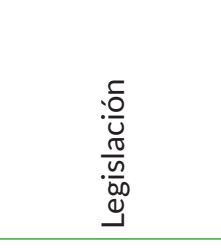 & 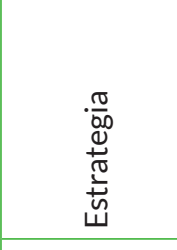 \\
\hline 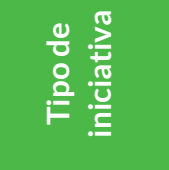 & $\frac{\pi}{\frac{0}{0}}$ & $\frac{\frac{\pi}{0}}{\frac{0}{3}}$ & 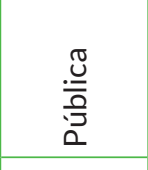 & $\frac{\pi}{\frac{0}{0}}$ & $\frac{\sqrt[0]{0}}{\frac{0}{0}}$ & $\frac{\pi}{\frac{0}{0}}$ \\
\hline 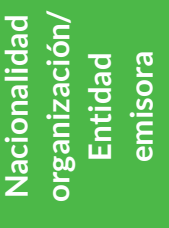 & 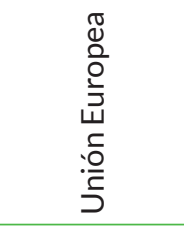 & 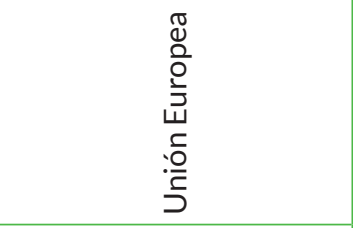 & 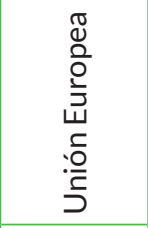 & 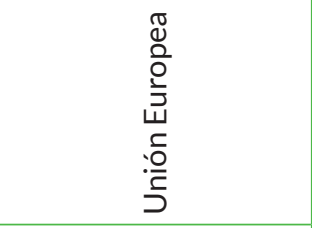 & 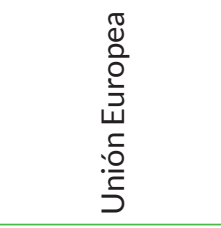 & 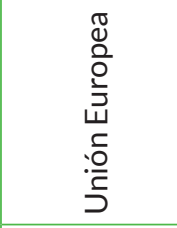 \\
\hline 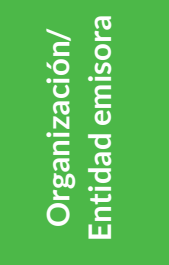 & 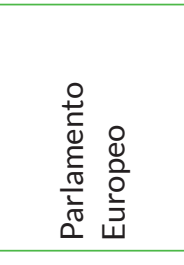 & 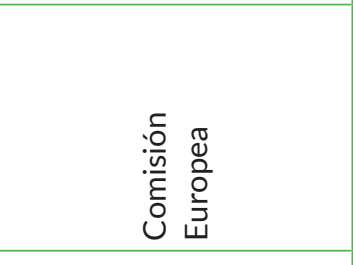 & 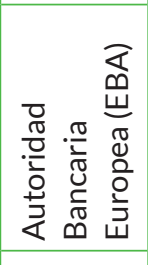 & 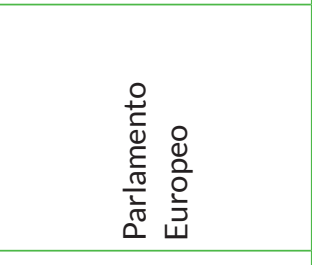 & 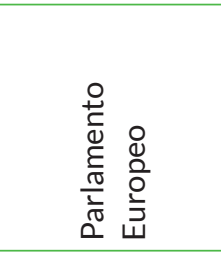 & 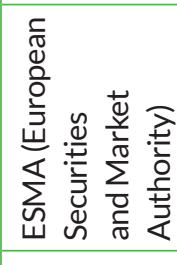 \\
\hline 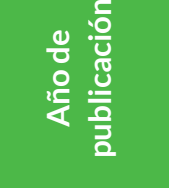 & $\stackrel{\infty}{\stackrel{\infty}{2}}$ & $\stackrel{\text { مे }}{\text { مे }}$ & ڤे̀े & مे & 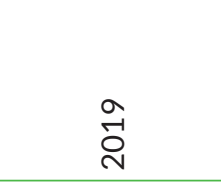 & 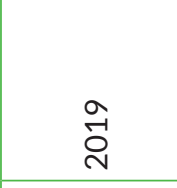 \\
\hline 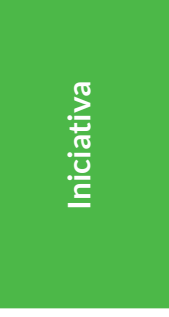 & 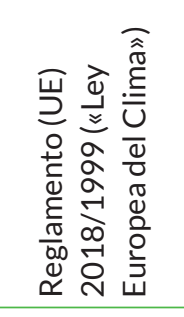 & 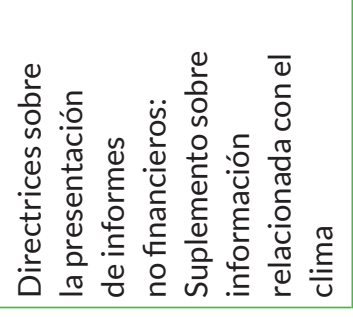 & 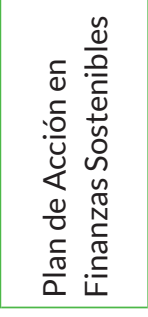 & 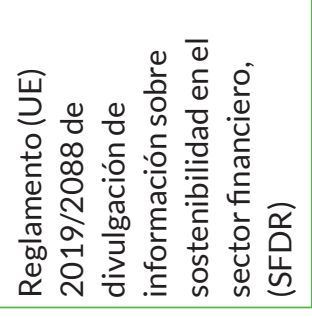 & 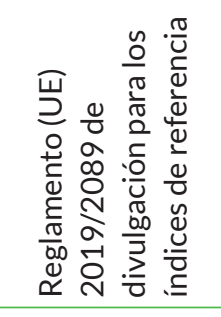 & 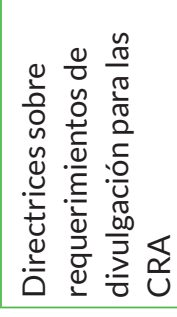 \\
\hline
\end{tabular}




\begin{tabular}{|c|c|c|c|c|c|c|c|c|}
\hline 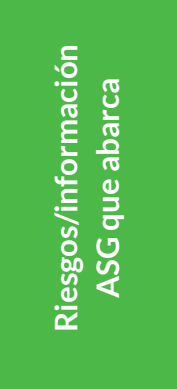 & 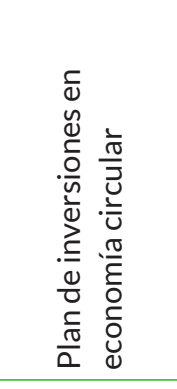 & 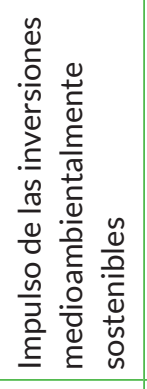 & 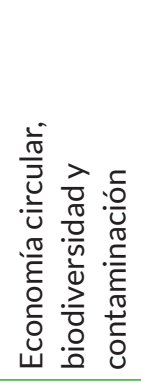 & 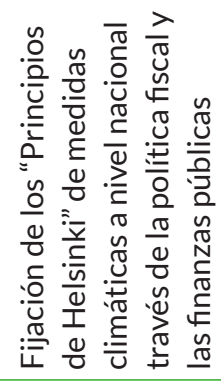 & 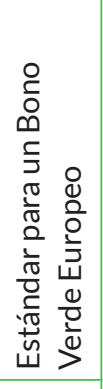 & 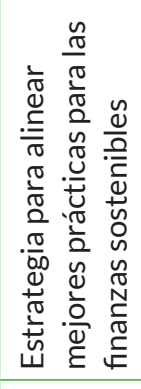 & 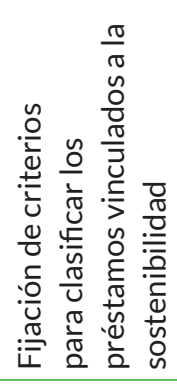 & 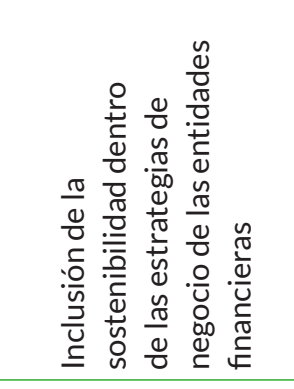 \\
\hline 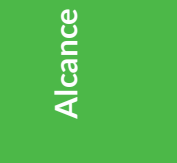 & 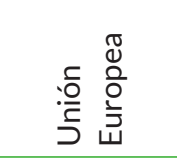 & $\begin{array}{l}\bar{\pi} \\
\frac{0}{0} \\
v\end{array}$ & $\begin{array}{l}\bar{\pi} \\
\frac{0}{0} \\
\frac{0}{0}\end{array}$ & $\begin{array}{l}\bar{\pi} \\
\frac{0}{0} \\
\end{array}$ & 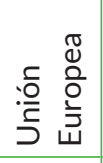 & $\begin{array}{l}\bar{\pi} \\
\frac{0}{0} \\
\end{array}$ & $\begin{array}{l}\bar{\pi} \\
\frac{0}{0} \\
0\end{array}$ & $\begin{array}{l}\bar{\pi} \\
\frac{0}{0} \\
v\end{array}$ \\
\hline 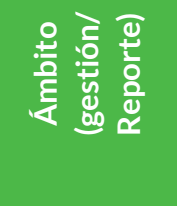 & 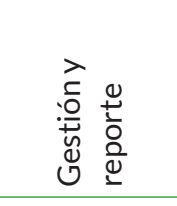 & 竞 & 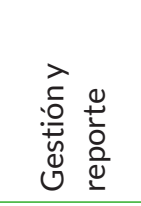 & 高 & 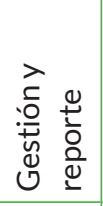 & 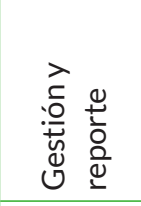 & 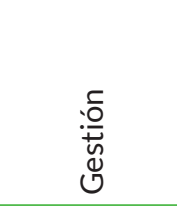 & 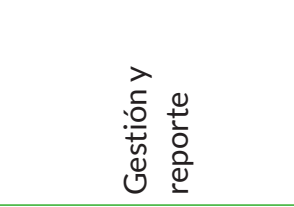 \\
\hline 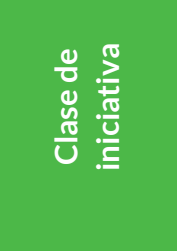 & $\begin{array}{l}\frac{\pi}{40} \\
\stackrel{0}{0} \\
\overline{0} \\
\vdots\end{array}$ & $\begin{array}{l}\frac{\pi}{4} \\
\stackrel{0}{0} \\
\overline{0} \\
\vdots\end{array}$ & 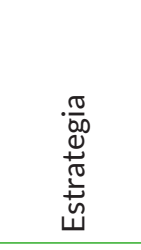 & 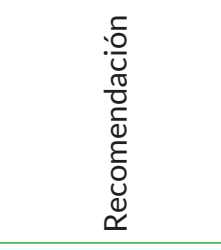 & 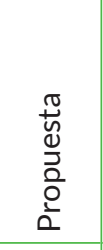 & 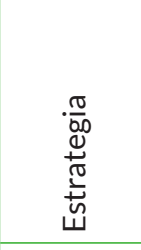 & 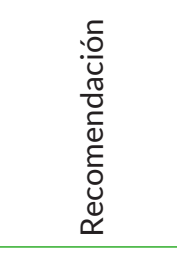 & 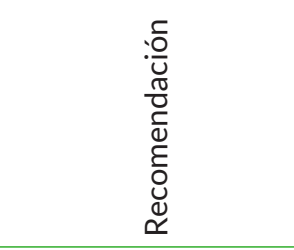 \\
\hline 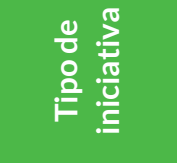 & $\frac{\sqrt[\pi]{0}}{\frac{0}{3}}$ & $\frac{\sqrt[\pi]{0}}{\frac{0}{3}}$ & 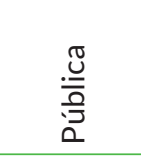 & $\frac{\frac{\pi}{2}}{\frac{0}{2}}$ & 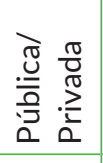 & $\frac{.00}{\frac{\pi}{3}}$ & 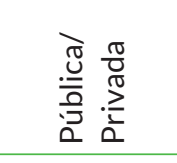 & 胥 $\frac{\pi}{\frac{\pi}{0}}$ \\
\hline 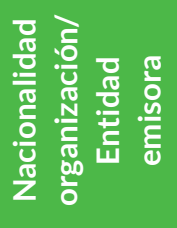 & 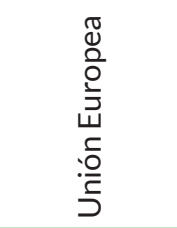 & 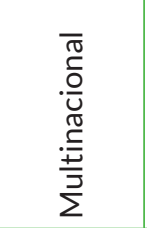 & 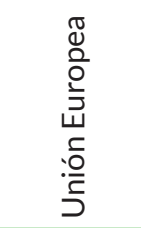 & 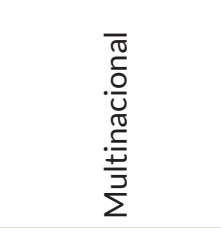 & 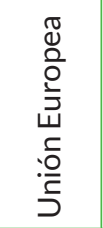 & 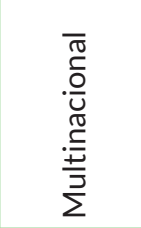 & 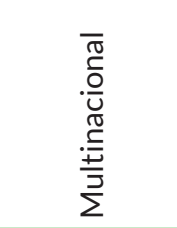 & 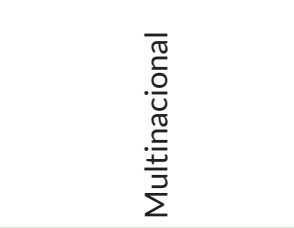 \\
\hline 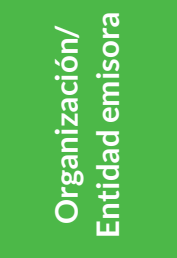 & 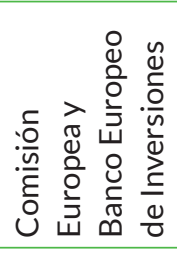 & 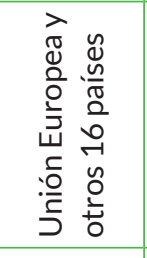 & 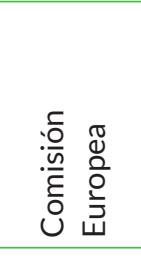 & 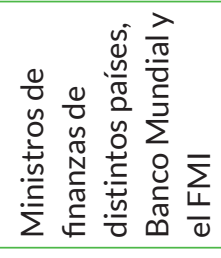 & 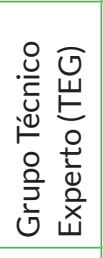 & 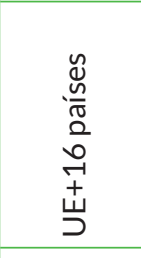 & 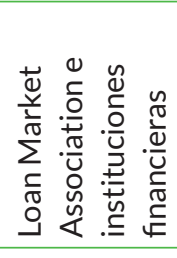 & 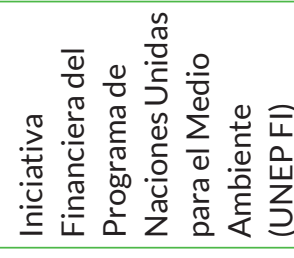 \\
\hline 这皆 & $\stackrel{\circ}{\stackrel{\sim}{2}}$ & ণ্े & مे & $\stackrel{\circ}{\stackrel{े}{2}}$ & مे & 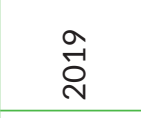 & 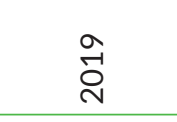 & مे \\
\hline 晜 & 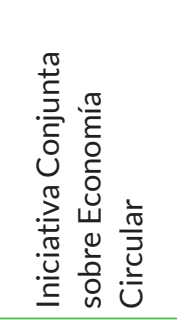 & 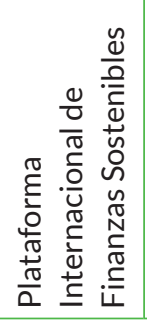 & 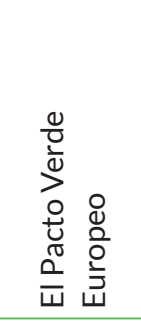 & 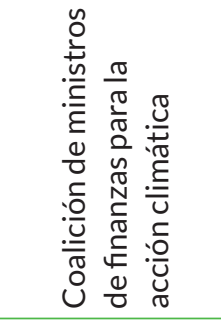 & 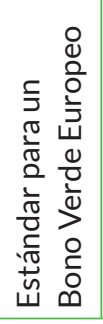 & 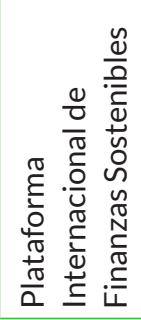 & 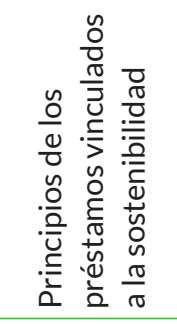 & 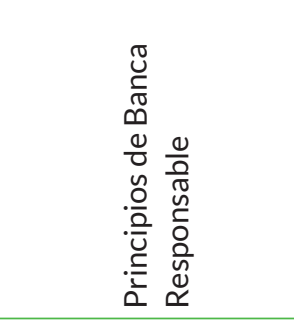 \\
\hline
\end{tabular}




\begin{tabular}{|c|c|c|c|c|c|c|}
\hline 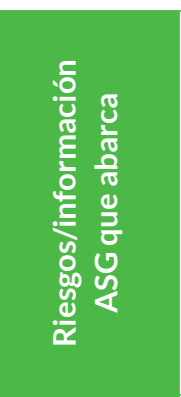 & 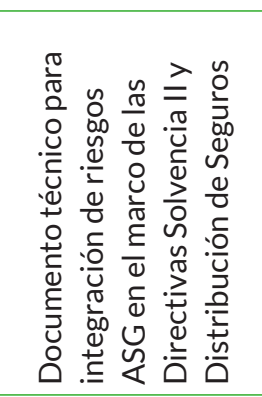 & 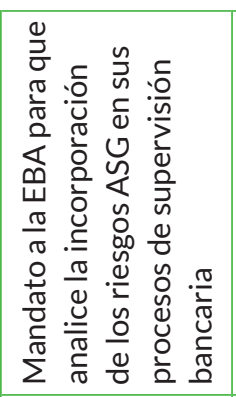 & 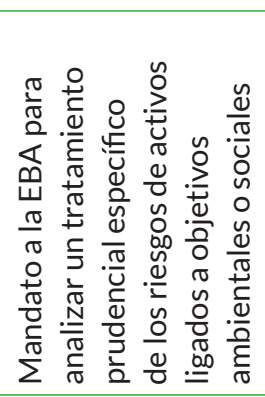 & 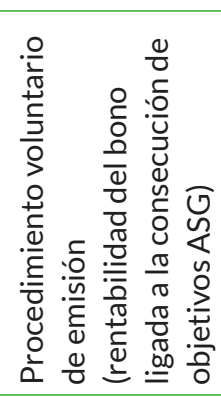 & 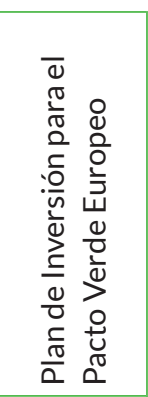 & 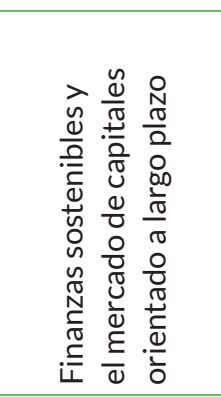 \\
\hline 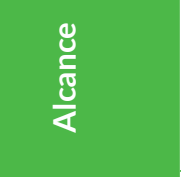 & 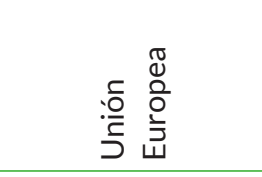 & 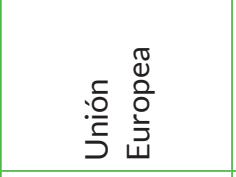 & שั & $\begin{array}{l}\bar{\pi} \\
\frac{0}{0} \\
\frac{0}{0}\end{array}$ & שั & : \\
\hline 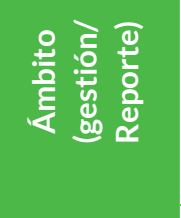 & 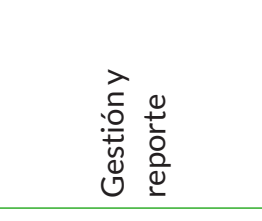 & 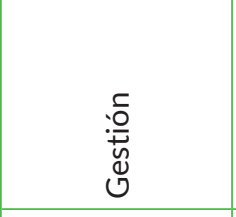 & 竞 & 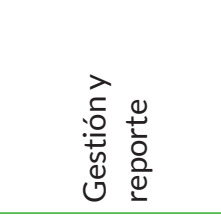 & 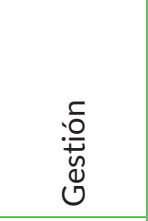 & 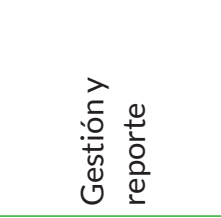 \\
\hline 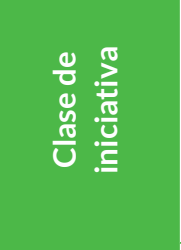 & 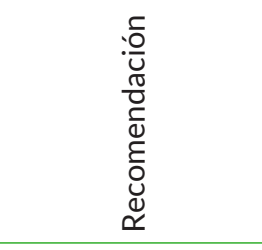 & 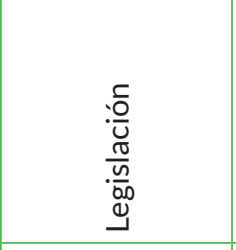 & 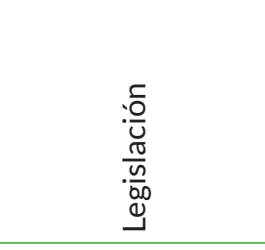 & 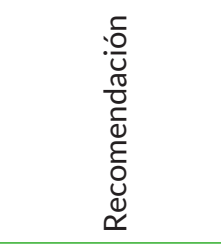 & $\begin{array}{l}\frac{\pi}{40} \\
\frac{0}{2} \\
\frac{0}{0} \\
\frac{0}{2}\end{array}$ & 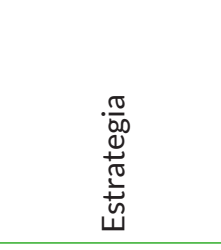 \\
\hline 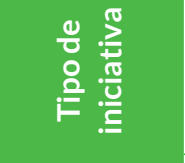 & $\frac{.00}{\frac{0}{a}}$ & $\frac{.00}{\frac{0}{3}}$ & $\frac{.00}{\frac{0}{3}}$ & 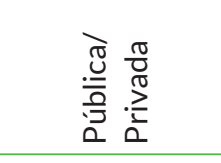 & $\frac{\pi}{\frac{\pi}{0}}$ & 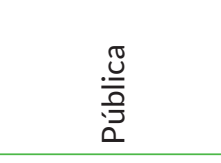 \\
\hline 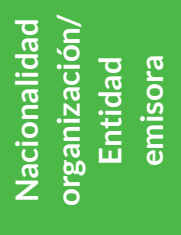 & 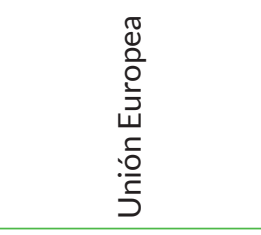 & 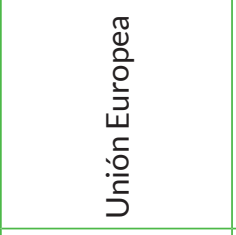 & 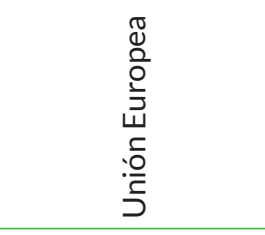 & 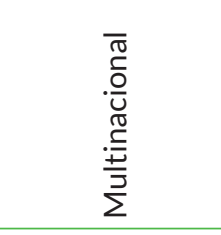 & 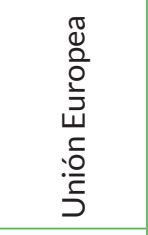 & 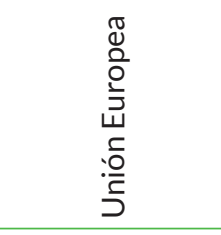 \\
\hline 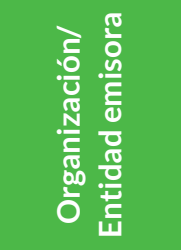 & 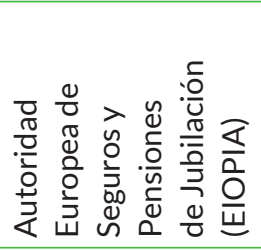 & 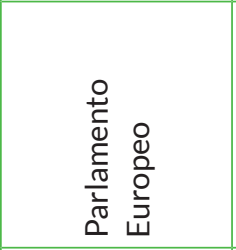 & 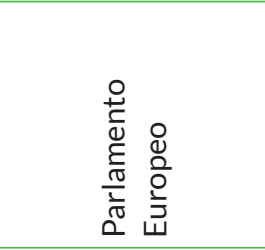 & 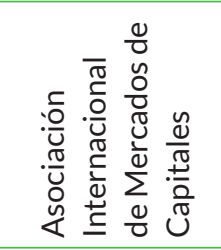 & 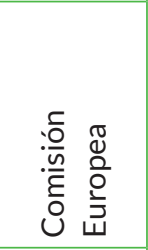 & 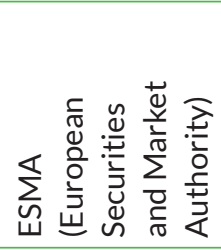 \\
\hline 突范 & $\stackrel{\vec{\nu}}{\vec{i}}$ & $\stackrel{\text { ¿े }}{\text { ¿े }}$ & $\stackrel{\text { ¿े }}{\text { ¿े }}$ & ণ্ণ & ণิ & ণิ \\
\hline & 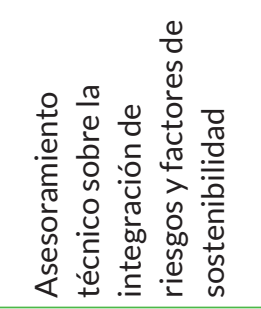 & 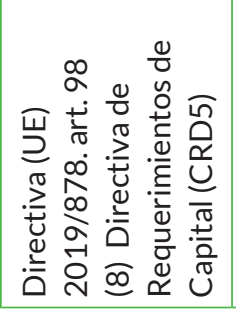 & 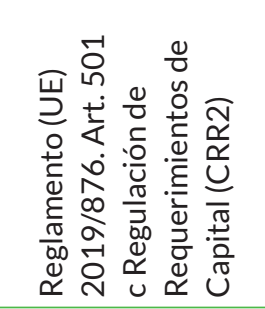 & 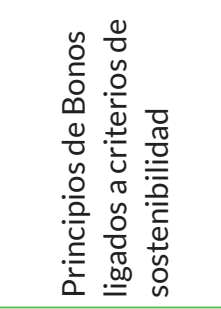 & 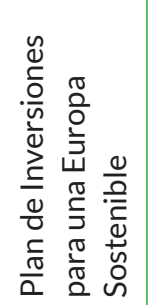 & 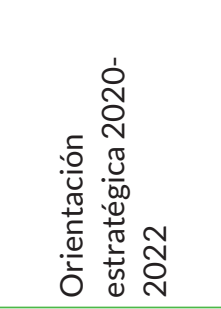 \\
\hline
\end{tabular}




\begin{tabular}{|c|c|c|c|c|c|c|c|}
\hline 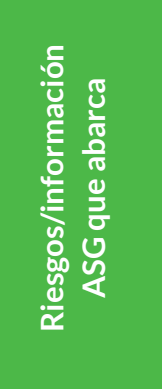 & 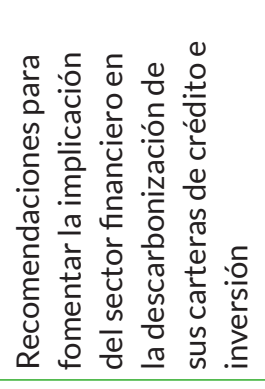 & 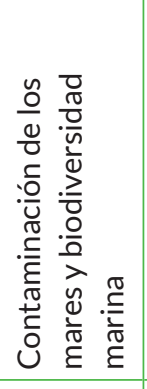 & 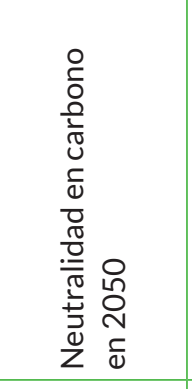 & 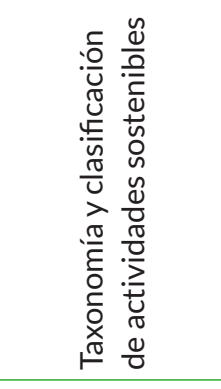 & 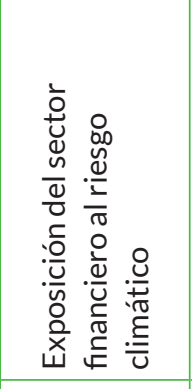 & 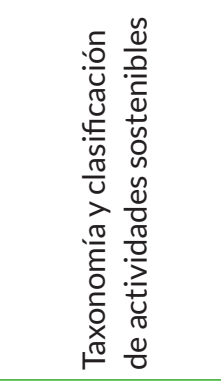 & 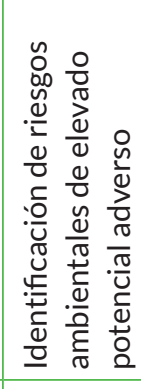 \\
\hline $\begin{array}{l}\frac{\mathscr{J}}{\mathrm{E}} \\
\frac{\mathrm{J}}{4} \\
\frac{\pi}{4}\end{array}$ & $\begin{array}{l}\bar{\pi} \\
\frac{0}{0} \\
\end{array}$ & 总 & 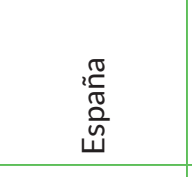 & 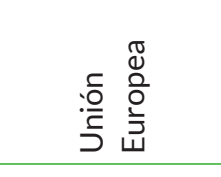 & $\begin{array}{l}\bar{\pi} \\
\frac{0}{0} \\
\end{array}$ & 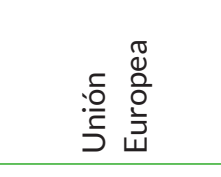 & $\begin{array}{l}\bar{\pi} \\
\frac{0}{0} \\
v\end{array}$ \\
\hline 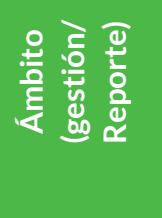 & 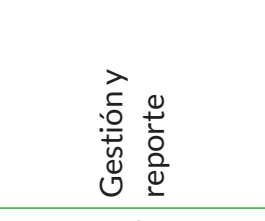 & : & 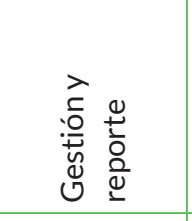 & 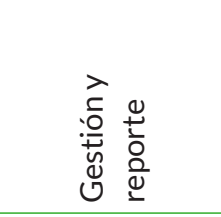 & 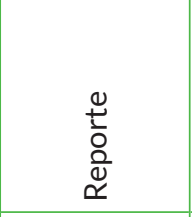 & 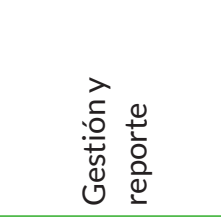 & 竞 \\
\hline 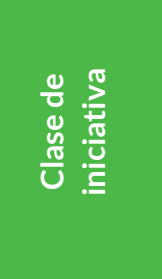 & 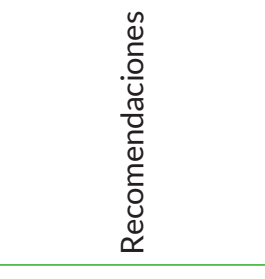 & $\begin{array}{l}\frac{\pi}{40} \\
\frac{0}{0} \\
\frac{0}{0} \\
0\end{array}$ & 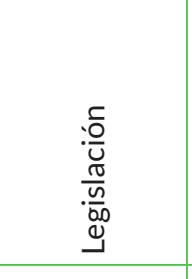 & 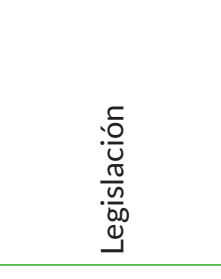 & 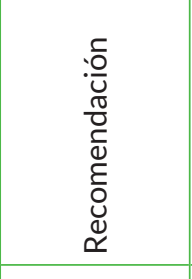 & 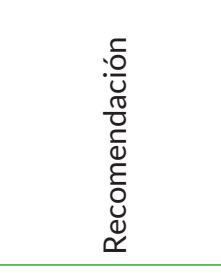 & 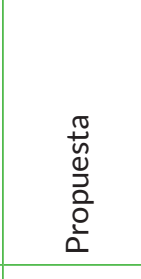 \\
\hline 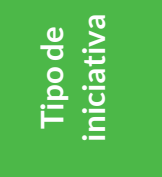 & 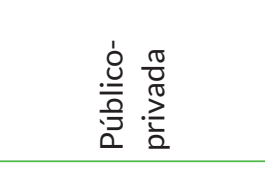 & $\frac{\sqrt[\pi]{0}}{\frac{0}{3}}$ & $\frac{\sqrt[3]{0}}{\frac{0}{3}}$ & $\frac{\sqrt[0]{0}}{\frac{0}{3}}$ & 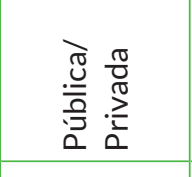 & $\frac{\sqrt[3]{0}}{\frac{30}{2}}$ & $\frac{\pi}{\frac{0}{\bar{O}}}$ \\
\hline 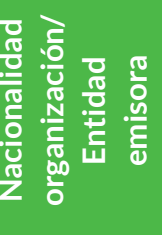 & 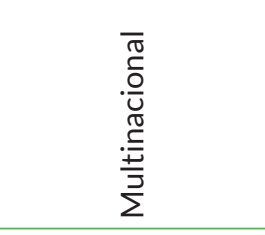 & 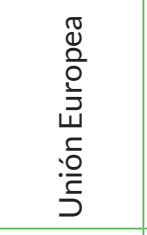 & 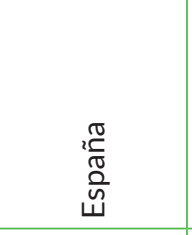 & 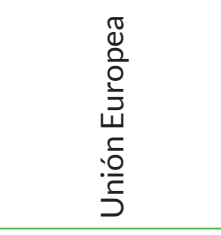 & 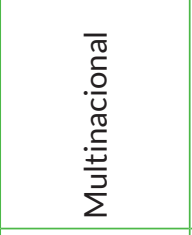 & 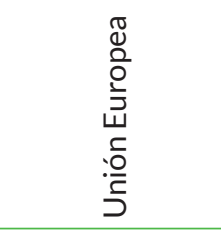 & 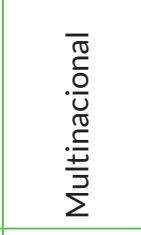 \\
\hline 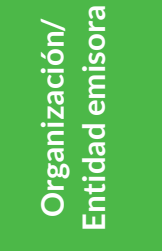 & 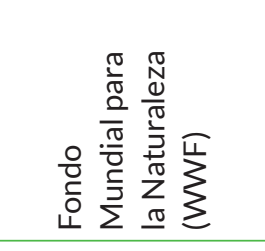 & 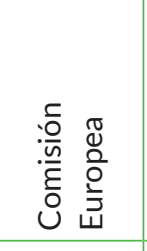 & 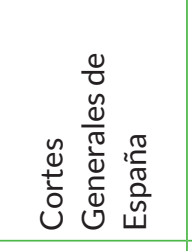 & 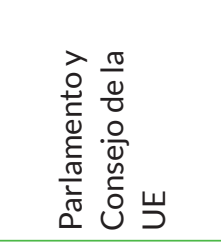 & 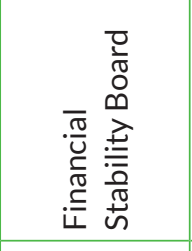 & 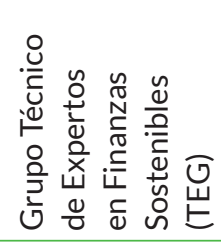 & 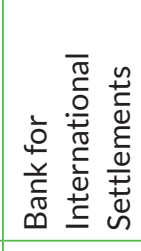 \\
\hline 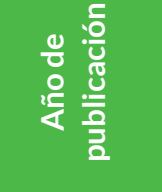 & 오 & 으 & 으 & 尺्ָे & ণ্ণ & ్ㅗ․ & 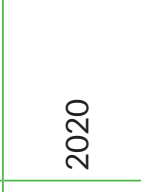 \\
\hline 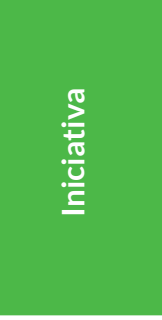 & 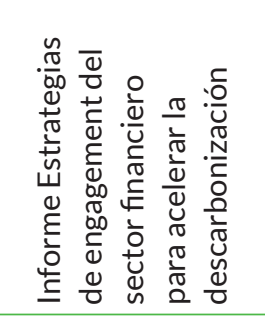 & 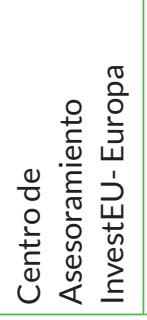 & 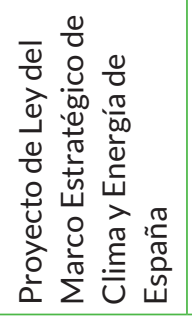 & 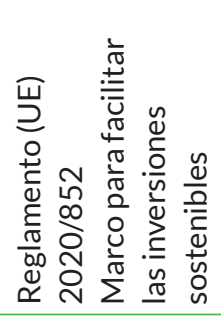 & 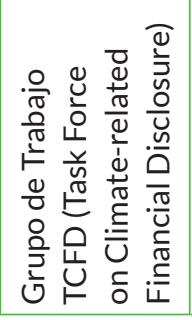 & 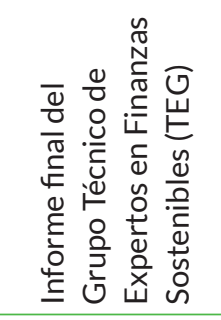 & 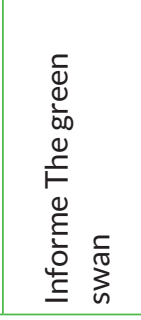 \\
\hline
\end{tabular}




\begin{tabular}{|c|c|c|c|c|c|c|}
\hline 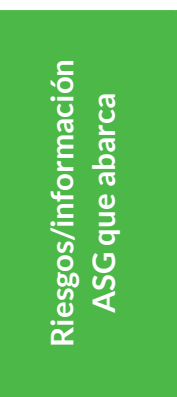 & 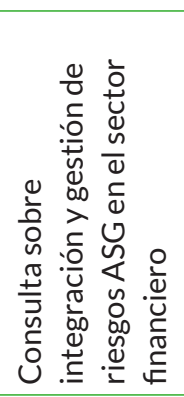 & 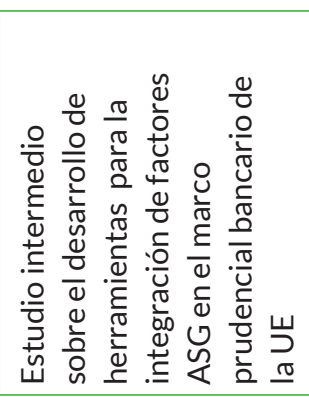 & 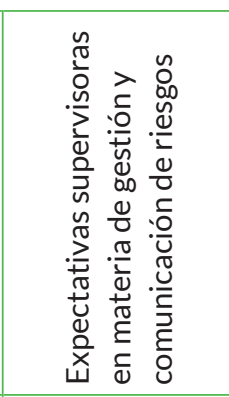 & 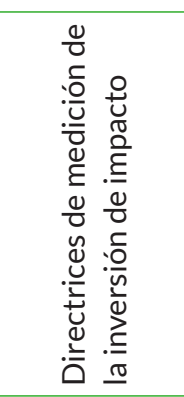 & 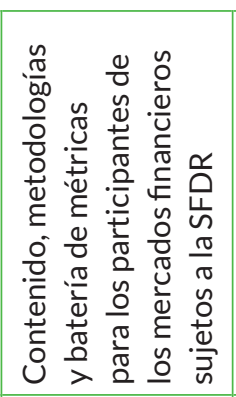 & 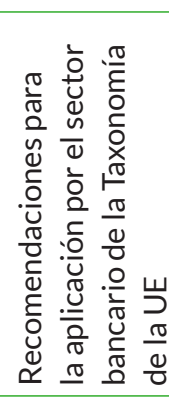 \\
\hline 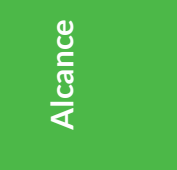 & 淧 & 次 & : & $\begin{array}{l}\bar{\pi} \\
\frac{0}{0} \\
\cup\end{array}$ & 苋 & $\begin{array}{l}\bar{\pi} \\
\frac{0}{0} \\
\end{array}$ \\
\hline 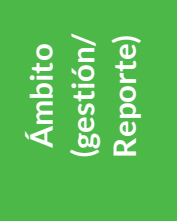 & 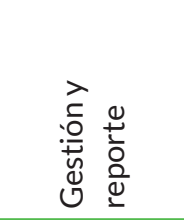 & 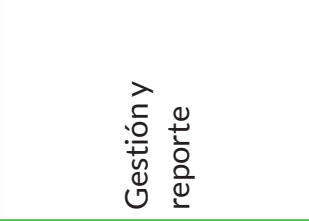 & 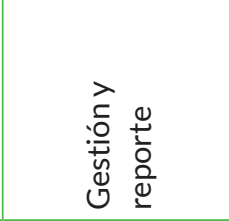 & 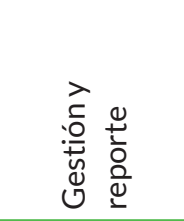 & 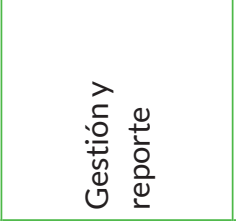 & 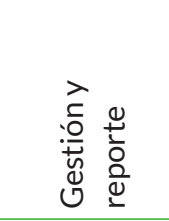 \\
\hline 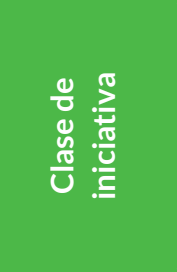 & $\begin{array}{l}\frac{\pi}{5} \\
\frac{\vec{n}}{5} \\
0 \\
0\end{array}$ & $\begin{array}{l}\frac{ㅇ}{\bar{y}} \\
\stackrel{\vec{J}}{\tilde{\omega}}\end{array}$ & 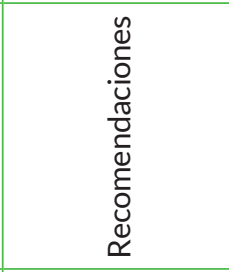 & 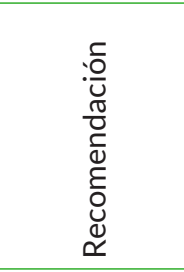 & 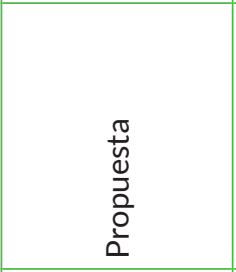 & 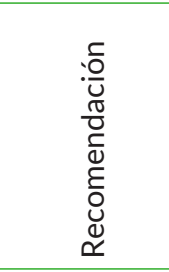 \\
\hline 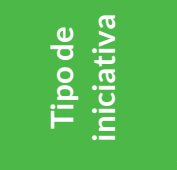 & $\frac{\frac{\pi}{2}}{\frac{3}{3}}$ & 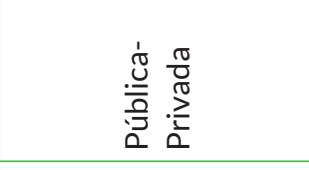 & 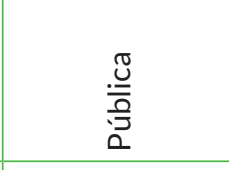 & $\frac{\sqrt[\pi]{0}}{\frac{.0}{3}}$ & $\frac{\frac{\pi}{0}}{\frac{0}{3}}$ & 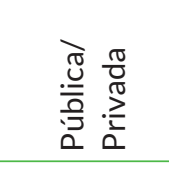 \\
\hline 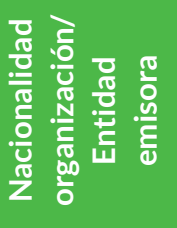 & 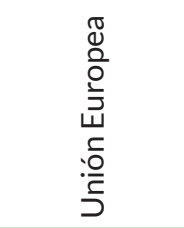 & 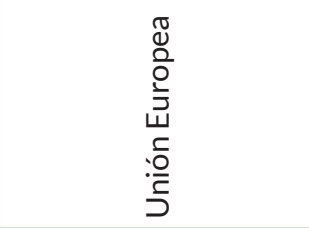 & 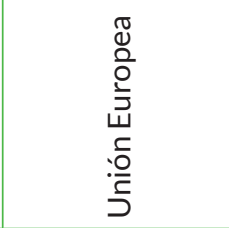 & 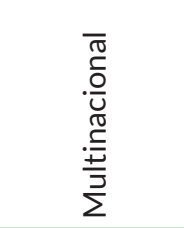 & 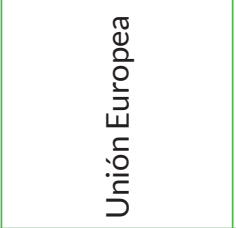 & 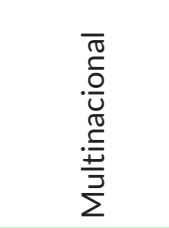 \\
\hline 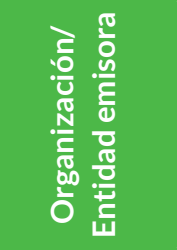 & 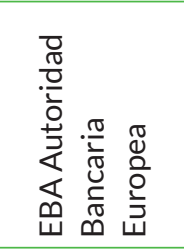 & 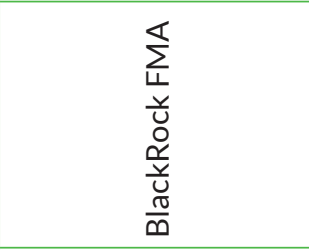 & 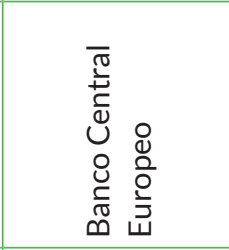 & 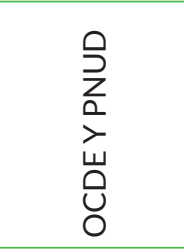 & 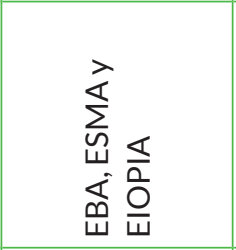 & 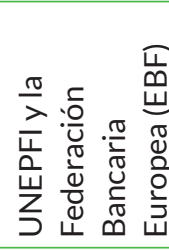 \\
\hline 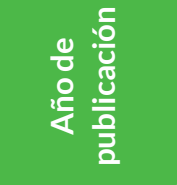 & 오 & ণิ & ণิ & ત્ત & ત્ત & ત્ત \\
\hline 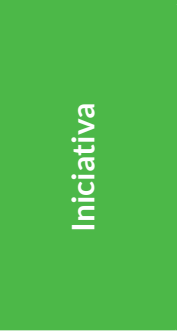 & 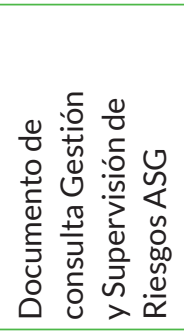 & 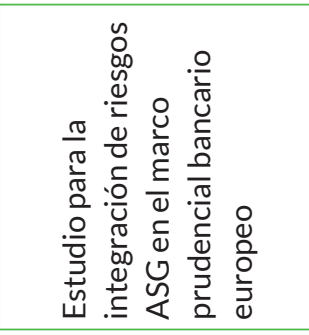 & 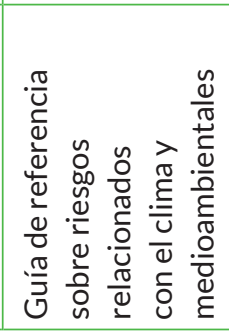 & 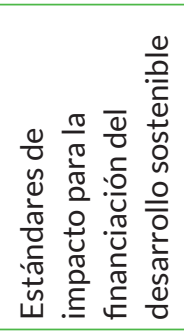 & 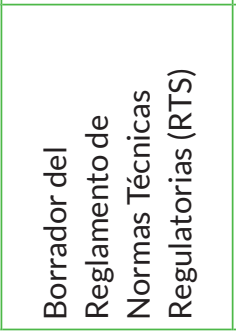 & 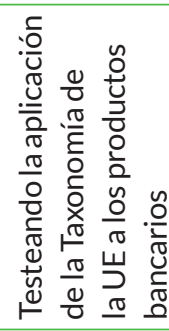 \\
\hline
\end{tabular}

